\title{
Self-tolerance curtails the B cell repertoire to microbial epitopes
}

\author{
Akiko Watanabe, ${ }^{1}$ Kuei-Ying Su, ${ }^{1,2}$ Masayuki Kuraoka, ${ }^{1}$ Guang Yang, ${ }^{1}$ Alexander E. Reynolds, ${ }^{1}$ \\ Aaron G. Schmidt, ${ }^{3,4}$ Stephen C. Harrison, ${ }^{5,6}$ Barton F. Haynes, ${ }^{1,7,8}$ E. William St. Clair, ${ }^{1,8}$ \\ and Garnett Kelsoe ${ }^{1,7}$ \\ 'Department of Immunology, Duke University School of Medicine, Durham, North Carolina, USA. ${ }^{2}$ Tzu Chi University \\ and Tzu Chi Medical Center, Hualien, Taiwan. ${ }^{3}$ Deparment of Microbiology, Children's Hospital, Harvard Medical School, \\ Boston, Massachusetts, USA. ${ }^{4}$ Ragon Institute of MCH, MIT and Harvard, Cambridge, Massachusetts, USA. 5 Laboratory of \\ Molecular Medicine, Children's Hospital, Harvard Medical School, Boston, Massachusetts, USA. ${ }^{6}$ Howard Hughes Medical \\ Institute, Boston, Massachusetts, USA. ${ }^{7}$ Duke Human Vaccine Institute and ${ }^{8}$ Department of Medicine, Duke University, \\ Durham, North Carolina, USA.
}

Immunological tolerance removes or inactivates self-reactive B cells, including those that also recognize cross-reactive foreign antigens. Whereas a few microbial pathogens exploit these "holes" in the B cell repertoire by mimicking host antigens to evade immune surveillance, the extent to which tolerance reduces the $B$ cell repertoire to foreign antigens is unknown. Here, we use single-cell cultures to determine the repertoires of human B cell antigen receptors (BCRs) before (transitional B cells) and after (mature B cells) the second B cell tolerance checkpoint in both healthy donors and in patients with systemic lupus erythematosus (SLE) . In healthy donors, the majority $(\sim 70 \%)$ of transitional B cells that recognize foreign antigens also bind human self-antigens (foreign+self), and peripheral tolerance halves the frequency of foreign+self-reactive mature B cells. In contrast, in SLE patients who are defective in the second tolerance checkpoint, frequencies of foreign+self-reactive $B$ cells remain unchanged during maturation of transitional to mature $B$ cells. Patterns of foreign+self-reactivity among mature B cells from healthy donors differ from those of SLE patients. We propose that immune tolerance significantly reduces the scope of the BCR repertoire to microbial pathogens and that cross-reactivity between foreign and self epitopes may be more common than previously appreciated.

Conflict of interest: The authors have declared that no conflict of interest exists.

Copyright: (c) 2019 American Society for Clinical Investigation

Submitted: June 4, 2018 Accepted: April 11, 2019 Published: May 16, 2019

Reference information: /CI Insight. 2019;4(10):e122551. https://doi. org/10.1172/jici.nsight.122551.

\section{Introduction}

The random recombination of $V, D$, and $J$ Ig gene segments produces a highly diverse repertoire of B cell antigen receptors (BCRs). While this process enables the generation of humoral responses against a wide range of harmful microorganisms, it often generates autoreactive BCRs. Indeed, about $75 \%$ of early immature human B cells are self-reactive, as determined by the generation of recombinant Abs (rAbs) from single cells $(1,2)$

During the transition from surface $\mathrm{Ig}^{-}$early immature B cells to surface $\mathrm{Ig}^{+}$immature B cells in bone marrow, the first tolerance checkpoint removes the majority of polyreactive $\mathrm{B}$ cells and/or those reactive with nuclear antigens $(1,2)$. The second tolerance checkpoint occurs during the transition from new emigrant (transitional 2 [T2]) B cells to mature, naive B cells and acts to remove or inactivate self-reactive B cells that have escaped the first checkpoint (1-4). During this transition, the frequency of self-reactive B cells is halved as determined by reactivity with a human cell line (HEp-2) $(1,5)$. Defects in both the first and second tolerance checkpoints have been linked to the development of autoimmune diseases, including systemic lupus erythematosus (SLE) and rheumatoid arthritis (RA) (5-8).

When tolerance removes or inactivates self-reactive B cells, those B cells that also recognize foreign epitopes that structurally resemble determinants on self-antigens are also lost. This action creates "holes" in the B cell repertoire that are exploited by microbial pathogens (9). Indeed, some pathogens, e.g., Campylobacter jejuni (10) and HIV-1 (11-13), are known to take advantage of these immunological holes by mimicking self-antigen and thereby mitigate effective control by the host immune system $(14,15)$. Despite these clinically important examples, the extent to which foreign specificities are lost at the tolerance checkpoints is unknown. To estimate the 
size and frequency of such repertoire holes, it is necessary to determine the reactivity of individual B cells against multiple self- and foreign antigens and then to compare the extent of self plus foreign cross-reactive specificities before and after tolerance checkpoints.

To survey for tolerance-induced holes in the human BCR repertoire before and after the second tolerance checkpoint, we cultured single human B cells on stromal cell layers that support B cell proliferation and differentiation to IgG-secreting plasmablasts and plasmacytes $(16,17)$. In this way, we obtained 2331 clonal IgGs from individual transitional and mature B cells representing the BCR repertoires before and after the second tolerance checkpoint $(1,2)$. These cells were recovered from the blood of healthy donors and from SLE patients who exhibit impairment of the second checkpoint $(2,5,6)$. We screened clonal IgGs against 12 human autoantigens and 8 foreign antigens in a multiplex bead (Luminex) assay and found that a high proportion of BCRs/clonal IgGs expressed by transitional B cells reacted with both foreign and self-antigens (F+S-reactive). The frequency of self-reactive IgGs, including F+S-reactive BCRs/clonal IgGs, decreased by half as transitional B cells entered mature B cell compartments in healthy controls. In contrast, SLE patients were significantly less efficient in the removal of F+S-reactive B cells. As a consequence of this impaired tolerance checkpoint, clonal IgGs expressed by mature B cells in SLE patients showed distinct patterns of reactivity against foreign antigens that were not present in healthy controls. These F+S-reactive BCRs typically had long heavy chain complementarity determining region 3 (HCDR3) and frequently utilized the $\mathrm{J}_{\mathrm{H}} 6$ gene segment, features shared by many broadly neutralizing Abs (bNAbs) against HIV-1 and influenza (18-22). We propose that a substantial fraction of BCR specificities that could offer broad protection against microbial pathogens is lost to immunological tolerance mechanisms.

\section{Results}

Single B cell cultures support robust proliferation and $\operatorname{IgG}$ production of human transitional and mature B cells. To characterize human $B$ cell repertoires before and after the second tolerance checkpoint $(1,2)$, we isolated single transitional B cells $\left(\mathrm{CD} 19^{+} \mathrm{CD} 27^{-} \mathrm{CD} 38^{\mathrm{hi}} \mathrm{CD} 10^{+} \mathrm{IgD}{ }^{+}\right)$and mature, naive $\mathrm{B}$ cells $\left(\mathrm{CD} 19^{+} \mathrm{CD} 27^{-} \mathrm{CD} 38^{\text {lo }} \mathrm{CD}^{-} 0^{-} \mathrm{IgD}^{+}\right)$from PBMCs of healthy donors (Supplemental Figure 1A; supplemental material available online with this article; https://doi.org/10.1172/jci.insight.122551DS1) (23), and cultured individual B cells in Nojima cultures, which support proliferation and plasmacytic differentiation of human B cells $(16,17)$. After 25 days of culture, we harvested culture supernatants containing clonal IgG produced by the differentiated progeny of single transitional or mature B cells and screened them on a panel of foreign and self-antigens $(17,24)$.

Both transitional and mature B cells proliferated robustly and secreted IgG with similar kinetics (Supplemental Figure 1, B and C). Single transitional B cells expanded about 300-fold by day 13, and then logarithmically increased until day 22 , when cell numbers approached a plateau; by day 25 , single transitional B cells had undergone 14-15 cell divisions to generate an average of 20,000 daughter cells (Supplemental Figure 1B). In some cultures, we could detect IgG in culture supernatants as early as day 13 , and by day $25 \mathrm{IgG}$ concentrations rose to approximately $3 \mu \mathrm{g} / \mathrm{ml}$ (Supplemental Figure 1C). Similarly, the proliferation of single mature B cells in culture produced approximately 50,000 progeny cells over 25 days of culture (Supplemental Figure 1B) and generated approximately $20 \mu \mathrm{g} / \mathrm{ml} \mathrm{IgG}$ in culture supernatants (Supplemental Figure 1C).

Single-cell cultures identify the second tolerance checkpoint in humans. Tolerance mechanisms remove or suppress self-reactive B cells in the bone marrow and periphery $(1,2)$. By analyzing rAbs synthesized from single B cells, Wardemann et al. (1) found that in healthy humans the proportion of self-reactive B cells drops by half ( $40 \%$ to $20 \%$ ) between "new emigrant" (transitional) and mature B cells, as determined by reactivity to a human cell line, HEp-2 (1). To determine general properties for recognition of specific selfand foreign antigens at the second tolerance checkpoint, we sorted transitional and mature B cells from 9 healthy donors into single-cell Nojima cultures and screened for reactivity of clonal IgGs against 12 self-antigens and 8 foreign antigens in a multiplex bead (Luminex) assay (Figure 1, A and B, and Supplemental Table 1) (17). Comparison of the frequencies of clonal IgGs that bound one or more self-antigens among transitional and mature B cell cultures revealed that in healthy donors, the proportion of self-reactive IgGs was reduced by half, from $26.8 \% \pm 11.3 \%$ in transitional B cells to $13.9 \% \pm 8.7 \%$ in mature B cells (Figure 1C). For all 9 healthy individuals, frequencies of self-reactive B cells decreased from the transitional stage to the mature B cell stage, with the frequency declining by $\geq 50 \%$ for 7 of 9 healthy donors (Figure 1D). This method of clonally expanding single B cells and multiplex screening with discrete foreign and self-antigens is sufficiently robust and sensitive to identify the second tolerance checkpoint in humans $(1,2,5)$. 
Peripheral tolerance removes foreign specificities from the $B$ cell repertoire. Tolerance can remove foreign specificity from the B cell repertoire when epitopes on foreign antigens structurally resemble those on self-antigens $(9,13,14)$ or when foreign reactivity is associated with polyreactive BCR (25-27). To assess the extent of the loss of foreign specificities from the transitional to mature B cell repertoire, we measured the reactivity of clonal IgGs to a defined set of foreign antigens, including HIV-1 gp41, gp140 (JR-FL), nitrophenyl acetyl-conjugated BSA (NP-BSA), OVA, keyhole limpet hemocyanin (KLH), anthrax recombinant protective antigen (rPA), streptavidin (SA), and influenza HAs (Figure 1, A and B, and Supplemental Table 1) and compared frequencies of clonal IgGs from transitional and mature B cell cultures that recognized epitopes within these foreign antigens (Figure 2).

In this cohort of healthy donors, frequencies of BCRs/clonal IgGs reactive with foreign antigens (F-reactive) fell significantly, by some $50 \%$, during the maturation of transitional to mature $\mathrm{B}$ cells, and the decrease was largely associated with reactivity to self-antigens $(F+S)$. Among F-reactive clonal IgGs, frequencies that bound both foreign and self-antigens ( $\mathrm{F}+\mathrm{S}$-reactive) decreased as transitional $\mathrm{B}$ cells matured in all 9 donors $(P<0.01$; Figure 2, A and D); in contrast, frequencies of clonal IgGs that were reactive with foreign but not self-antigens (F-only) did not significantly change (15\% reduction) between transitional and mature B cell cultures (Figure 2, B and D). The loss of $\mathrm{F}+\mathrm{S}$-reactive BCRs across the second tolerance checkpoint resulted in a significant reduction $(40 \%, P<0.05$; Figure $2, \mathrm{C}$ and $\mathrm{D})$ in F-reactive mature $\mathrm{B}$ cells, demonstrating that while the second tolerance checkpoint removes self-reactivity, it also incidentally depletes a significant fraction of F-reactive BCRs.

$F+S$-reactive $B$ cells are not purged in SLE patients. We next examined whether our single-cell approach was sensitive enough to uncover defective tolerance checkpoints in SLE patients $(2,5,6)$. We isolated transitional and mature B cells from PBMCs of 14 SLE patients, and determined the binding of clonal IgGs to foreign and self-antigens as described above (Figure 1 and Supplemental Figure 2). Consistent with previous reports $(2,5,6)$, frequencies of self-reactive IgGs expressed by B cells in patients with lupus was unchanged across the second tolerance checkpoint (Figure 3A). While the frequency of self-reactive BCRs decreased from transitional to mature B cells more than 2-fold in 7 of 9 (78\%) healthy individuals, it did so in only 1 of 14 (7\%) SLE patients (Figure 1D and Figure 3A). In half of the samples from SLE patients, the frequency of self-reactive IgGs increased from the transitional to mature B cell stage (Figure 3A), a feature not seen in any sample from healthy donors (Figure 1D). These results demonstrate that Nojima cultures reflect the defective second tolerance checkpoint in SLE patients identified by single-cell PCR methods $(2,5,6)$.

In contrast to the decrease in F-reactive and F+S-reactive clonal IgGs from transitional to mature $\mathrm{B}$ cells in healthy donors (Figure 2), these frequencies were unchanged during B cell maturation in SLE patients (Figure 3B and Supplemental Figure 2, C and D) consistent with impaired tolerance $(2,5,6)$ and the introduction of $\mathrm{F}+\mathrm{S}$-reactive $\mathrm{B}$ cells into the mature $\mathrm{B}$ cell pools.

Peripheral tolerance does not suppress all self-reactive B cells equally. It is unknown whether the second checkpoint removes all self-reactive B cells equally during the T2-to-mature transition, or instead removes B cells reactive to certain self-antigens more effectively than those reactive to others. To discriminate between these alternatives, we determined the percentage of clonal IgGs that bound each antigen among all $\mathrm{IgG}^{+}$ transitional and mature B cell cultures, respectively (Supplemental Table 1), and compared the ratio of the 2 stages (relative to transitional B cells; Figure 4A); given that the frequencies of IgGs binding to individual antigens were very low, we combined results from 9 healthy individuals (total $1083 \mathrm{IgG}^{+}$samples) and $14 \mathrm{SLE}$ patients (1248 $\mathrm{IgG}^{+}$samples). In healthy donors, frequencies of antigen-binding IgGs decreased $\geq 2$-fold $(P<0.01)$ during the maturation of transitional to mature B cells for most of the self-antigen panel, though the decrease was modest (20\%-35\%) for B cells that bound RNP, Scl-70, and Jo-1. The frequencies of dsDNA- and kynureninase-binding (KYNU-binding) IgGs were little changed or even increased (50\%) across the second checkpoint (Figure 4A). In contrast to healthy donors, in SLE patients frequencies of autoreactive clonal IgGs for most of the panel of self-antigens tested were unchanged or nonsignificantly diminished ( $<40 \%$ reduction) across the second checkpoint (Figure $4 \mathrm{~B}$ ). We note specifically that frequencies of clonal IgGs that bound Sm, dsDNA, and KYNU increased $\geq 2$-fold as transitional B cells matured in SLE patients (Figure 4B). Taken together, our results indicate that not all self-reactive B cells - within the avidity limits of our Luminex assay and our sample sizes — are equally likely to be removed by the mechanisms of peripheral tolerance.

We next carried out a similar analysis for each foreign antigen in our screening panel (Figure 4, $\mathrm{C}$ and D). For most foreign antigens, frequencies of antigen-binding IgGs decreased by about $40 \%$ as 
A

Healthy

Transitional

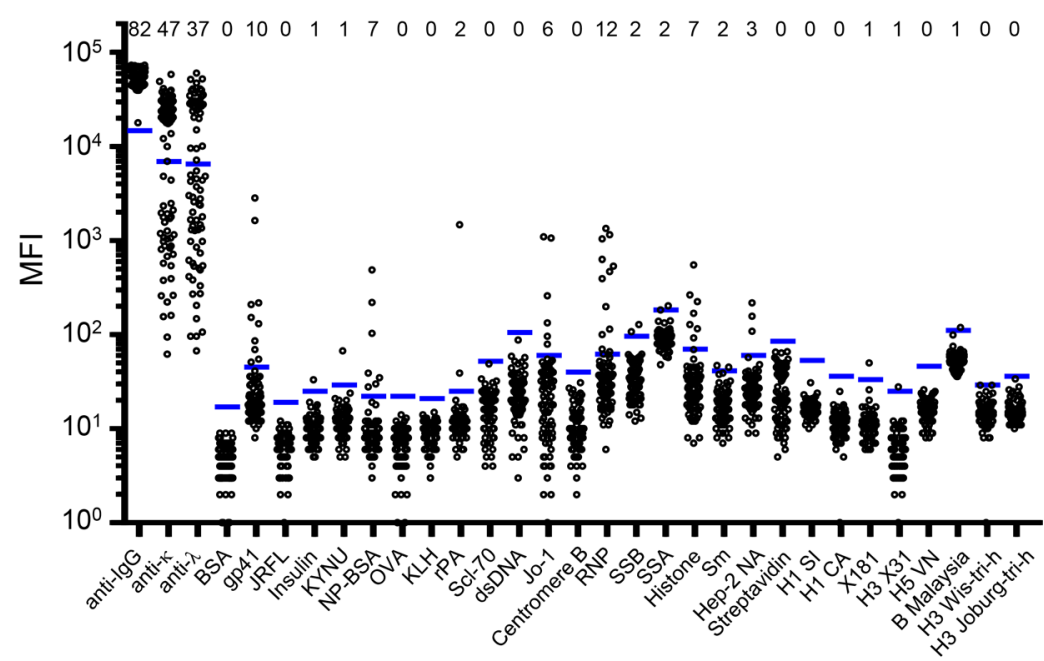

B

Healthy

Mature

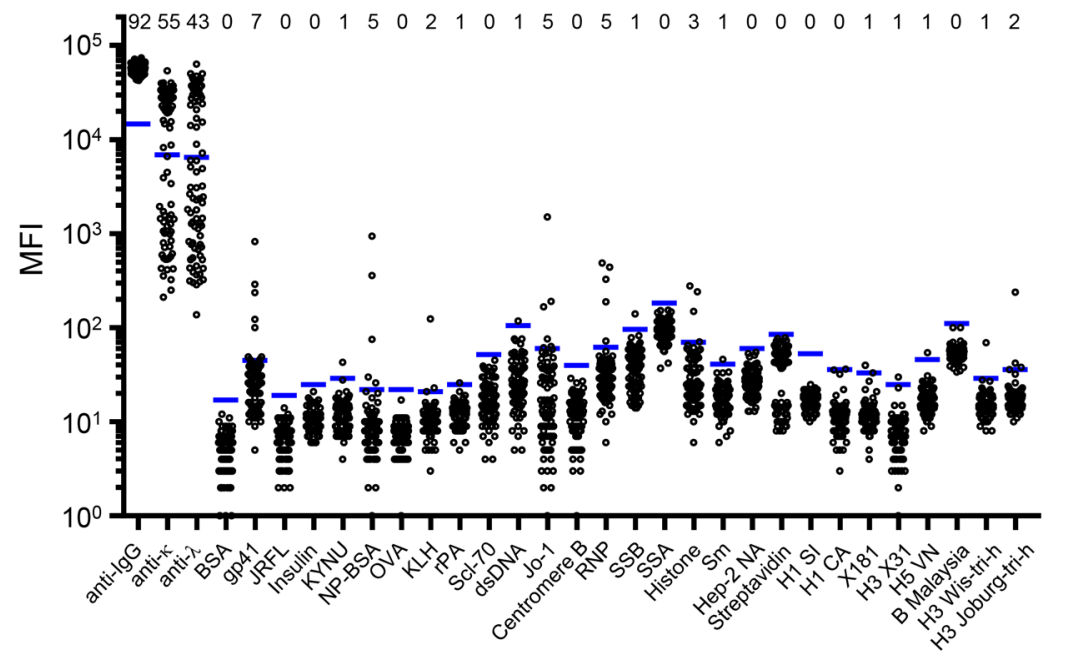

C

Healthy

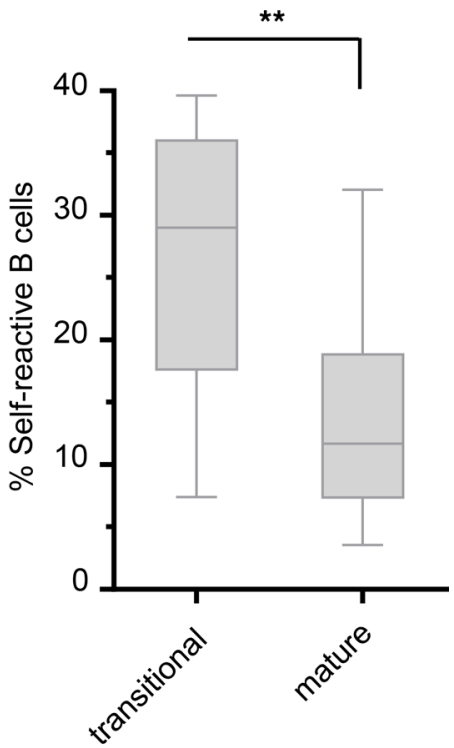

D

Healthy

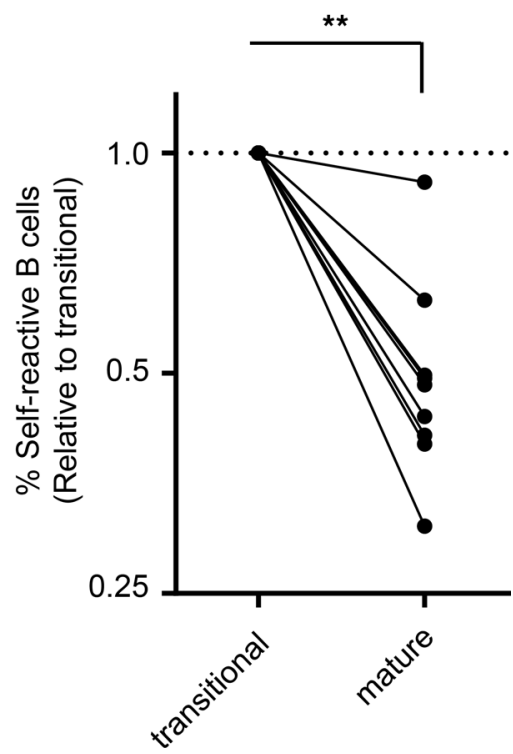

Figure 1. Peripheral tolerance removes self-reactive mature B cells in healthy donors. Reactivity of clonal Abs in culture supernatants of individual single $B$ cell cultures ( $n=429$ from transitional B cell cultures and $n=654$ from mature B cell cultures) from healthy individuals were tested against 4 positive and negative controls (anti-lgG, anti-lgא, anti-lg $\lambda$, and BSA) and a panel of self- and foreign antigens by Luminex multiplex assays. (A and B) Representative diagrams for transitional B cells (A) and mature B cells (B) from healthy donors. Each dot represents an individual test for each antigen. Figures represent $n=82$ and $n=92$ for transitional B and mature B cells, respectively. Blue bars indicate the threshold median fluorescence intensities (MFI) for each antigen (average + 6SD of B cellnegative, mock-treated samples). Number of positive reactions is indicated for each antigen above each panel. We tested subsets ( $n=145$ and $n=213$ for transitional and mature B cells, respectively) of culture supernatant IgGs for binding to HAs. (C and $\mathbf{D}$ ) Frequency of self-reactive B cells in transitional and mature B cells $(n=9)$. (C) Boxes extend from the 25 th to 75 th percentile, and lines in the boxes represent medians. Error bars represent minimum to maximum values. (D) Each dot represents an individual subject. For each individual, frequencies of self-reactive B cells were normalized with those of transitional $B$ cells, and transitional and mature $B$ cells are connected with lines. ${ }^{* *} P<0.01$ determined by Wilcoxon's matched-pairs signed-rank test.

transitional B cells matured in healthy donors, with the exception of SA-binding B cells (Figure 4C). Frequencies of SA-binding IgGs rose nearly 2-fold across the second checkpoint (Figure 4C). By contrast, the frequencies of F-reactive IgGs expressed by B cells in SLE patients were unchanged from the transitional to the mature stage for most of the foreign antigens (Figure 4D), except IgGs binding to SA or HIV-1 envelope gp140 (JR-FL), which rose substantially during the transition (Figure 4D). These results 
A
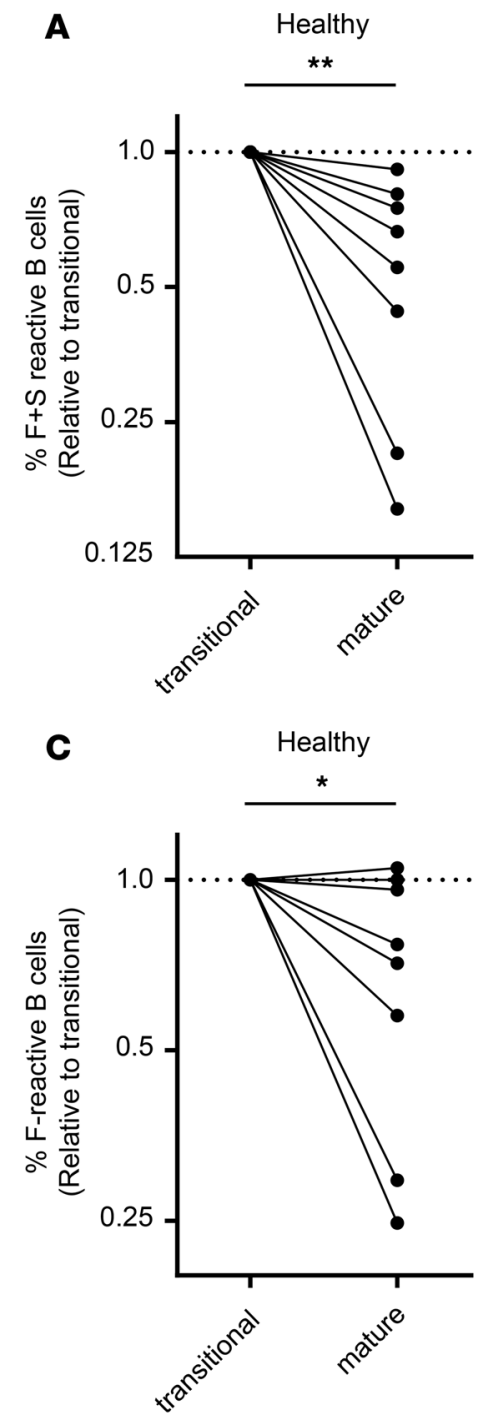

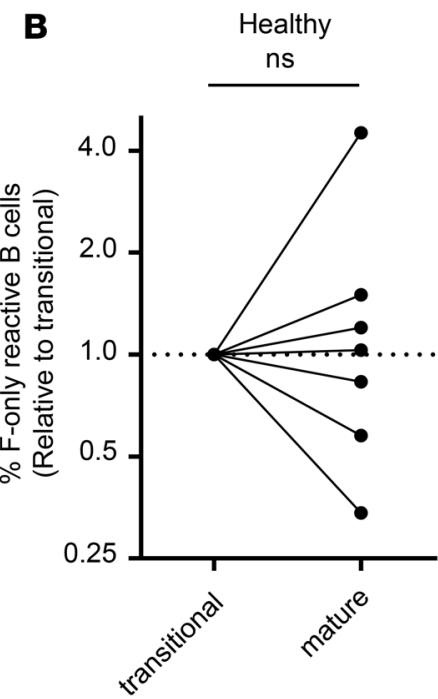

D Healthy

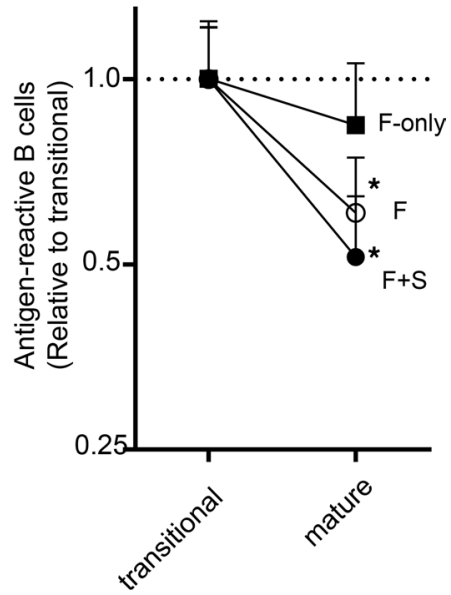

Figure 2. Peripheral tolerance removes $\mathbf{F + S}$-reactive $B$ cells. Frequencies of clonal Abs that react with one or more foreign antigens in transitional and mature B cell cultures (see legend of Figure 1). Foreign antigen-reactive Abs in C were divided by their reactivity with self-antigens, and the frequencies of $F+S$-reactive $A$ bs $(\mathbf{A})$ and F-only-reactive Abs (B) are shown. In A-C, each dot represents an individual subject. For each individual, frequencies of self-reactive Abs were normalized with those of transitional B cells, and transitional and mature B cells are connected with lines. In B, connected lines were not generated for those individuals whose transitional and/or mature B cell cultures did not contain F-only-reactive Abs. ${ }^{*} P<0.05$, ${ }^{* *} P<0.01$, NS $P>0.05$ determined by Wilcoxon's matched-pairs signed-rank test (2-tailed). (D) Frequency of F-reactive (open circles), F+S-reactive (filled circles), and F-only-reactive (filled squares) $B$ cells in transitional and mature $B$ cells are connected with lines. For each group, frequencies of individual samples were normalized to the average frequency of antigen-binding B cells among transitional B cells. ${ }^{*} P<0.05$ determined by Wilcoxon's matched-pairs signed-rank test (2-tailed) to compare antigen-reactive B cells in transitional $B$ cells and mature B cells for each group.

suggest that epitopes recognized by $\mathrm{F}+\mathrm{S}$-reactive $\mathrm{BCRs}$ are present in many foreign antigens, including the protective antigen (PA) of Bacillus anthracis, influenza HA, KLH, and even hen egg OVA. The second tolerance checkpoint removes B cells reactive to such epitopes from the mature B cell pool in healthy donors but not in SLE patients.

Mature B cells in SLE patients show distinct foreign reactivity. As F+S-reactive B cells provide paratopic specificities distinct from those provided by F-only B cells, we reasoned that the preimmune BCR repertoire of F-reactive, mature B cells would be different if tolerance controls were incomplete. Indeed, SLE patients with defective B cell tolerance checkpoints $(1,2,5,6)$, produce more avid anti-influenza Abs than healthy controls after vaccination (28) and can generate broadly neutralizing HIV-1 Abs that cross-react with DNA (27). 

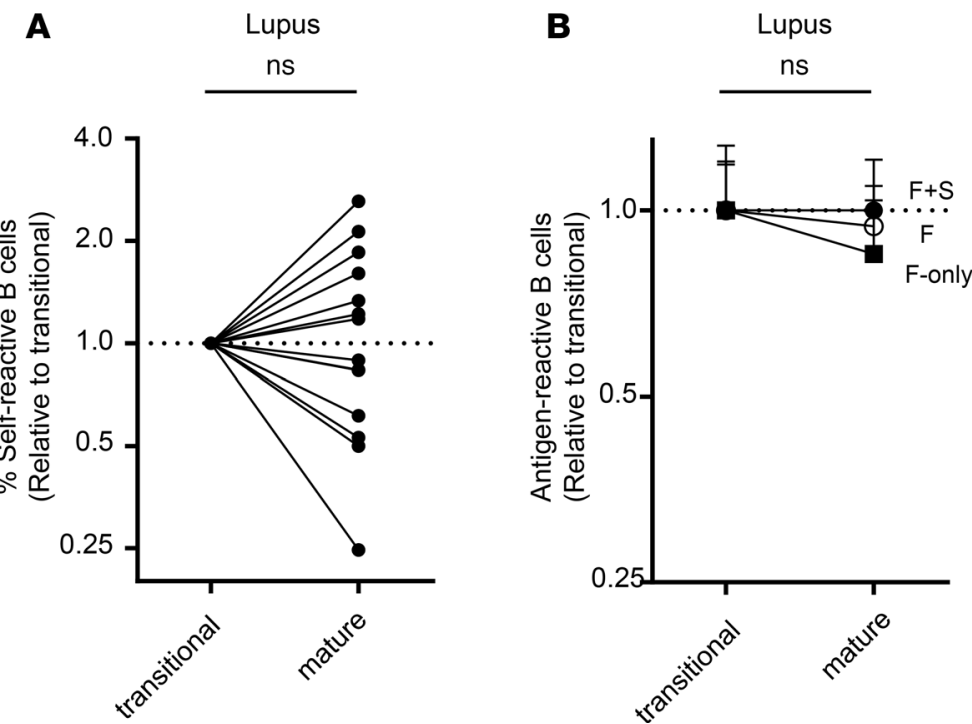

Figure 3. Removal of self-reactive mature B cells is impaired in SLE patients. Reactivity of clonal Abs in culture supernatants of individual single B cell cultures $(n=506$ from transitional B cell cultures and $n=742$ from mature $B$ cell cultures) from individuals with lupus were tested by Luminex multiplex assays (see also Supplemental Figure 2). (A) Frequency of self-reactive B cells in transitional and mature B cells in SLE patients are shown. For each individual, frequencies of self-reactive $B$ cells were normalized with those of transitional $B$ cells, and transitional and mature $B$ cells are connected with lines. NS: $P>0.05$ determined by Wilcoxon's matched-pairs signed-rank test (2-tailed). (B) Frequency of F-reactive (open circles), F+S-reactive (filled circles), and F-only-reactive (filled squares) B cells in transitional and mature $B$ cells are connected with lines. For each group, frequencies of individual samples were normalized with the average frequency of antigen-binding B cells in transitional B cells. NS $P>0.05$ determined by Wilcoxon's matched-pairs signed-rank test (2-tailed).

To examine whether defective tolerance checkpoints that do not remove F+S-reactive mature B cells in SLE patients could establish a substantially distinct naive BCR repertoire for foreign antigens, we compared distribution of BCR reactivity between healthy donors and SLE patients. For both foreign and self-antigens, we determined the percentage of antigen-binding IgGs (no. reactions for each antigen/no. total reactions for all antigens) within SLE patients and healthy individuals, and then took the ratio of these percentages (SLE patients relative to healthy controls; Figure 5). As before, we combined results from 9 healthy donors and 14 SLE patients to determine the overall binding distributions of the mature B cell repertoire in healthy donors and SLE patients. We found that IgGs expressed by mature B cells in SLE patients showed a distinct reactivity with both self-antigens $(P<0.01)$ and foreign antigens $(P<0.05)$. Compared with healthy controls, the IgGs expressed by mature B cells in SLE patients more frequently contained clones that bound both self- and foreign antigens, such as Sm, Scl-70, insulin, OVA, KLH, influenza HAs, and HIV-1 gp140 (JR-FL) (Figure 5, A and B). By contrast, distribution of BCR reactivity of transitional B cells did not significantly $(P>0.05)$ differ between donor types (Figure $5, C$ and $D)$. The primary BCR repertoires of SLE patients are substantially different from those of healthy controls, and that difference reflects the pruning of $B C R s$ that recognize epitopes shared by foreign and self-determinants.

Long HCDR3s are features of $F+S$-reactive $B C R$ s. To characterize the $\mathrm{V}_{\mathrm{H}} \mathrm{DJ}_{\mathrm{H}}$ gene signatures of $\mathrm{F}+\mathrm{S}$-reactive $B$ cells, we amplified $V_{H} D J_{H}$ rearrangements from cDNA synthesized from cell pellets of individual Nojima cultures that were $\operatorname{IgG}^{+}$, and compared $\mathrm{V}_{\mathrm{H}}, \mathrm{D}$, and $\mathrm{J}_{\mathrm{H}}$ gene usage and HCDR3 length among $4 \mathrm{~B}$ cell groups: (i) S-only-reactive (bind to self- but not foreign antigens), (ii) $\mathrm{F}+\mathrm{S}$-reactive, (iii) F-only-reactive, and (iv) unknown (did not react with any screening antigen). Among self-reactive groups (S-only and $\mathrm{F}+\mathrm{S}$ ), we chose to recover $\mathrm{V}_{\mathrm{H}} \mathrm{DJ}_{\mathrm{H}}$ rearrangements from samples that bound Sm and RNP in particular, as (i) they were most frequent in transitional and mature B cell cultures and (ii) anti-Sm Abs often coexist with anti-RNP Abs in SLE patients $(29,30)$. We obtained and sequenced $\mathrm{V}_{\mathrm{H}} \mathrm{DJ}_{\mathrm{H}}$ rearrangements from 42 S-only ( 42 of $96 ; 44 \%$ of all Sm- and/or RNP-reactive samples among S-only; $19 \%$ of the S-only-reactive group), $49 \mathrm{~F}+\mathrm{S}$ ( 49 of $98 ; 50 \%$ of all Sm- and/or RNP-reactive samples within the F+S-reactive group; $28 \%$ of the F+S-reactive group), $72 \mathrm{~F}$-only ( 72 of $97 ; 74 \%$ of the F-only-reactive group), and 67 unknown (67/1833; 4\% of the unknown group) cultures for analysis. 

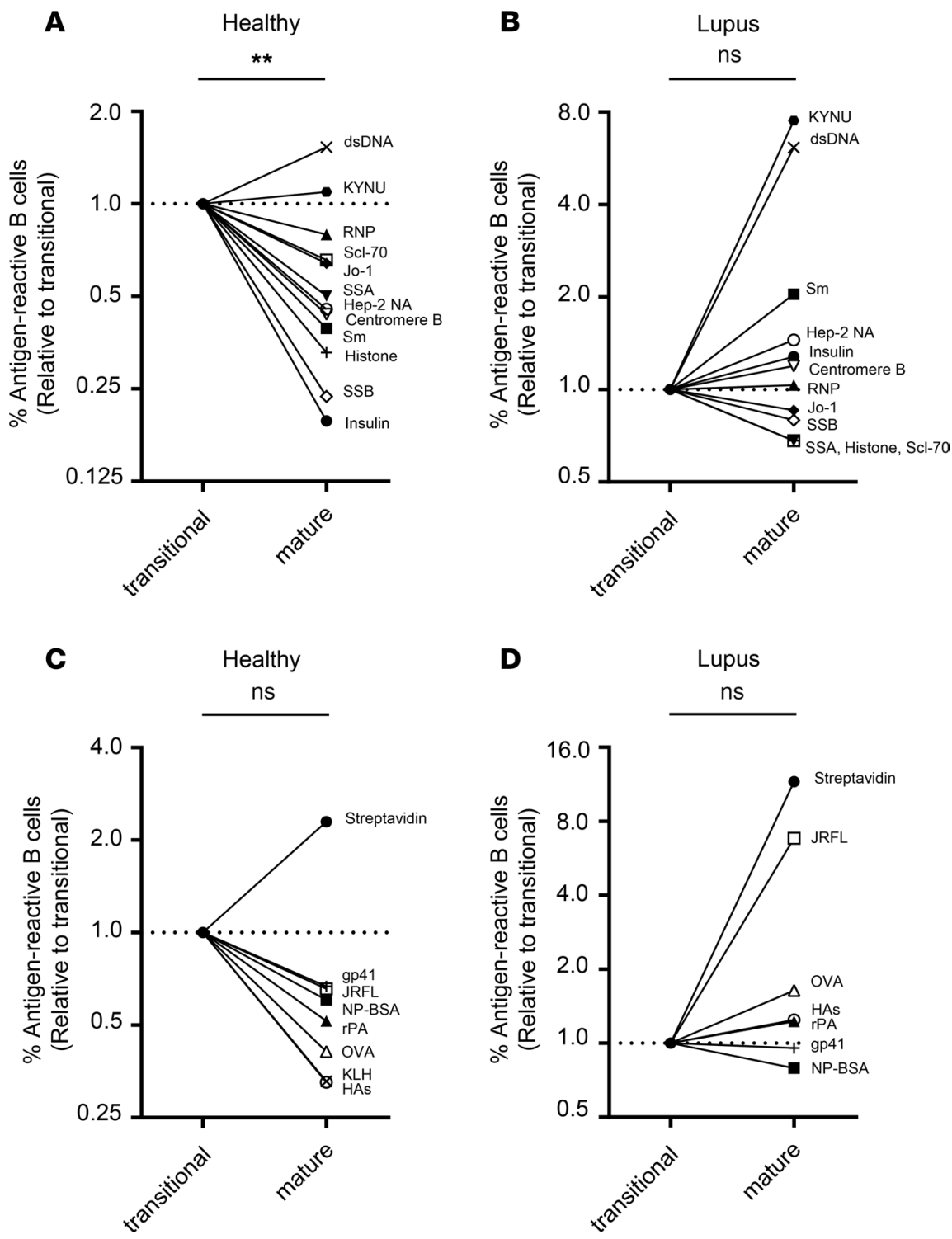

Figure 4. Peripheral tolerance removes $B$ cell specificities to many antigen types. Ab binding results of transitional and mature $\mathbf{B}$ cells in healthy donors ( $\mathbf{A}$ and $\mathbf{C}$ ) and SLE patients (B and $\mathbf{D})$ for individual self-antigens $(\mathbf{A}$ and $\mathbf{B})$ : Sm (filled square), Hep-2NA (open circle), insulin (filled circle), centromere B (open inverted triangle), histone (+), SSB (open diamond), SSA (filled inverted triangle), Jo-1 (filled diamond), Scl-70 (open square), RNP (filled triangle), dsDNA $(\times)$, KYNU (filled hexagon)]; and foreign antigens (C and D): gp41 (+), JR-FL (open square), KLH ( $\times$ ), HAs (open circle), rPA (filled triangle), SA (filled circle), OVA (open triangle), and NP-BSA (filled square). For each antigen, we calculated the frequency of antigen-binding Abs, and ratios of the frequencies of antigen-binding Abs (relative to Abs expressed by transitional B cells) within healthy donors ( $n=1083 \mathrm{lgG}$ Abs; see legend of Figure 1) and SLE patients ( $n=1248$ lgG Abs, see legend of Figure 3 and Supplemental Figure 2) are shown. We tested subsets ( $n=358$ and $n=416$ for healthy donors and SLE patients, respectively) of culture supernatant IgGs for binding to HAs. In each panel, connected lines were not generated when transitional and/or mature B cell cultures did not contain Abs that bound indicated antigens. ${ }^{* *} P<0.01$; NS: $P>0.05$ determined by Wilcoxon's matched-pairs signed-rank test (2-tailed).

Long HCDR3s were common features of the BCRs of self-reactive groups, as expected (1, 31-33). HCDR3 lengths were comparable $(P=0.38)$ in the S-only $(17.9 \pm 4.3$ aa $)$ and $\mathrm{F}+\mathrm{S}$-reactive groups $(18.7$ $\pm 4.9 \mathrm{aa})$, and these values were significantly higher $(P<0.001)$ than in the F-only $(15.3 \pm 4.1 \mathrm{aa})$ and unknown (14.6 $\pm 4.1 \mathrm{aa}$ ) groups (Figure $6 \mathrm{~A})$. Regardless of the B cell type (transitional or mature) or the donor type (healthy donors or lupus patients), self-reactive groups had greater HCDR3 length than did non-self-reactive groups (Supplemental Figure 3).

Although there were no obvious differences in $V_{H}$ and $D_{H}$ gene usage between self-reactive and non-self-reactive groups (Figure 6B), we found that the long HCDR3s in self-reactive BCR groups 
A

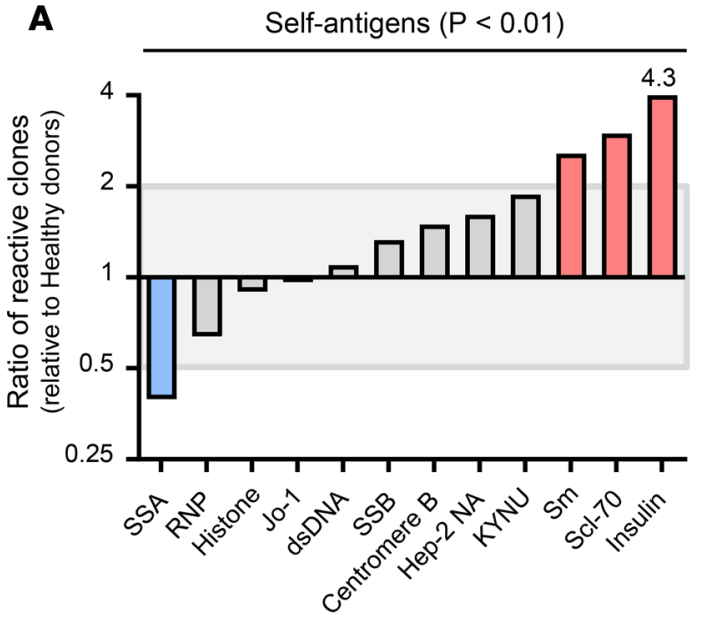

C

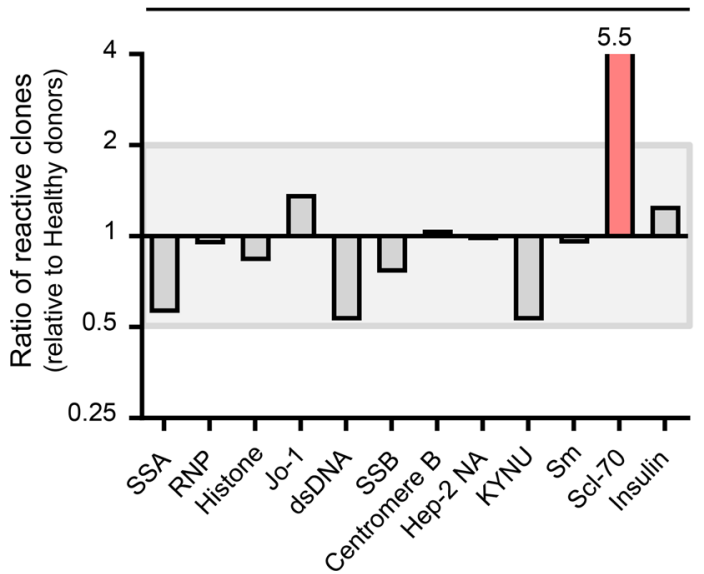

B Foreign antigens $(P<0.05)$

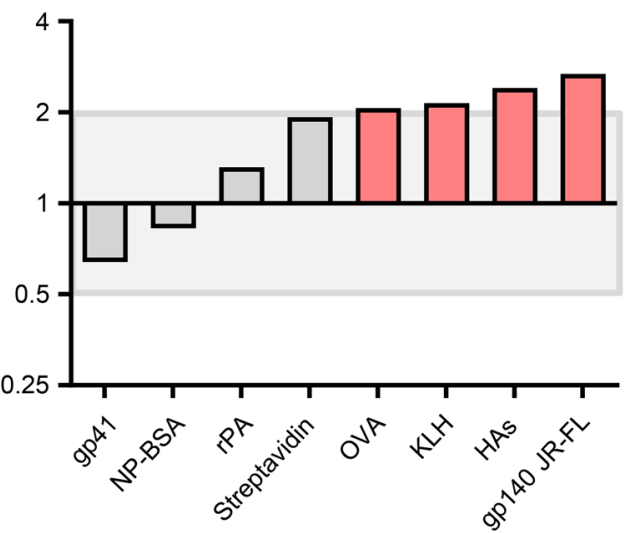

$\uparrow$

Frequent

in lupus

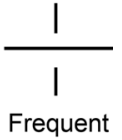

in healthy

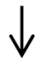

\section{D}

Foreign antigens $(P>0.05)$

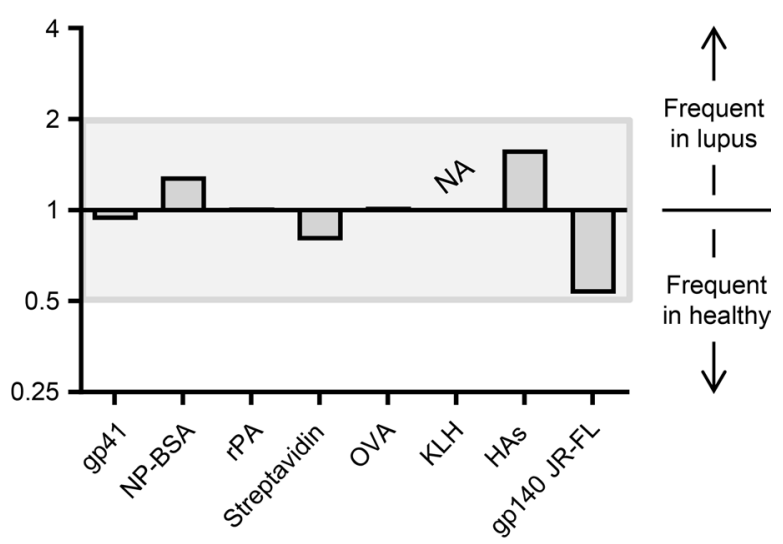

Figure 5. Mature, naive B cells in lupus patients exhibit distinct reactivity with foreign antigens. Ab binding results of total 429 and 654 lgG Abs (transitional and mature B cells, respectively) from 9 healthy individuals and 506 and 742 lgC Abs(transitional and mature B cells, respectively) from 14 individuals with lupus were combined, and distributions of the BCR reactivity of mature B cells ( $\mathbf{A}$ and $\mathbf{B})$ and of transitional $B$ cells (C and $\mathbf{D})$ compared between healthy and lupus individuals. For each antigen, we counted antigen-reactive Abs and added these numbers to set as $100 \%$; then frequencies of antigen-reactive Abs were determined for individual antigens. Ratios of the frequencies of antigen-reactive $B$ cells from individuals with lupus relative to healthy controls for self-antigens ( $\mathbf{A}$ and $\mathbf{C}$ ) and foreign antigens ( $\mathbf{B}$ and $\mathbf{D}$ ) are shown. Antigens given ratios of $\geq 2$ and $\leq 0.5$ are indicated with red and blue bars, respectively. Statistical significance was determined by $\chi^{2}$ independence test. NA indicates "not applicable," as we did not recover any KLH-binding cells from transitional $\mathbf{B}$ cells among patients with lupus. In $\mathbf{A}$ and $\mathbf{C}$, numbers above bars indicate actual values that exceed $y$ axis.

were linked to frequent $J_{H} 6$ gene usage (Figure 6C). In general, we recovered $V_{H} 3$ and $V_{H} 4$ genes, and $D_{H} 3$ genes most frequently from B cells in all groups (Figure 6B). By contrast, we found that the $J_{H} 4$ gene was most frequent in F-only-reactive (49\%) and unknown groups (54\%), while the $J_{H} 6$ gene was most frequent (55\%) in S-only and $\mathrm{F}+\mathrm{S}$-reactive groups (Figure 6B). Distributions of $J_{H}$ gene usage differed significantly $(P=0.002)$ between self-reactive (S-only and $\mathrm{F}+\mathrm{S}$-reactive) and non-self-reactive (F-only and unknown) groups (Figure 6C). Thus, self-reactive BCRs, including F+S-reactive BCRs, have long HCDR3s in association with frequent $J_{H} 6$ gene usage. Collectively, our results suggest that peripheral tolerance removes a substantial fraction of B cells that bear long HCDR3s, are autoreactive, and include a substantial population of transitional B cells that also recognize epitopes on microbial pathogens. The structural features of BCRs that are removed at the second tolerance checkpoint in humans are those often present in bNAbs for HIV-1 and influenza (18-22).

$F+S$-reactive IgGs bind self- and foreign antigens through proximal paratopic sites. To determine whether the $\mathrm{F}+\mathrm{S}$-reactive $\mathrm{BCR}$ recognize self- and foreign epitopes via overlapping paratopes, we carried out inhibition assays where we incubated $\mathrm{F}+\mathrm{S}$-reactive $\mathrm{IIgGs}$ with decreasing concentrations of soluble inhibiting (foreign) antigens and assessed their effect on rIgG binding to the same antigen (homologous inhibition) or reactive self-antigens (heterologous inhibition). 
A

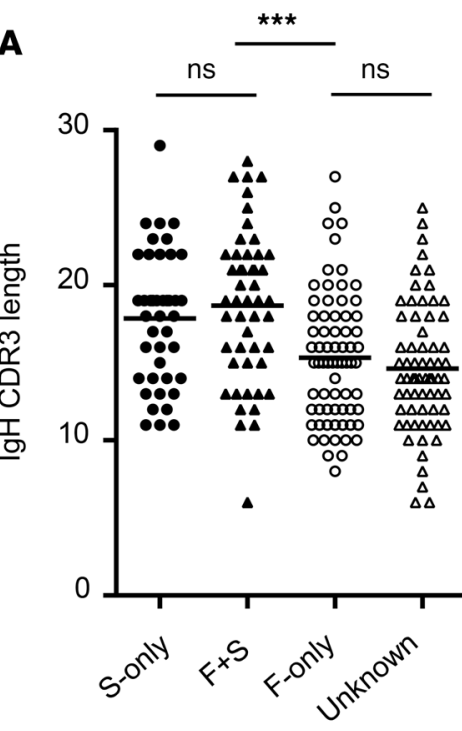

B
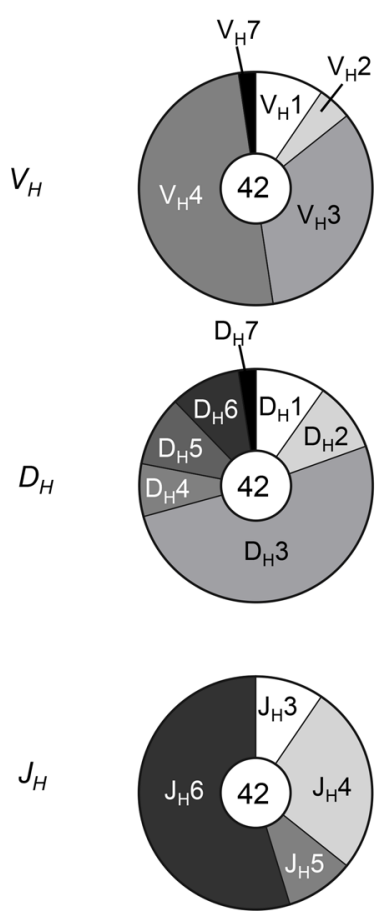

C

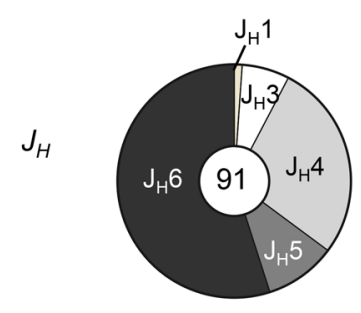

Self

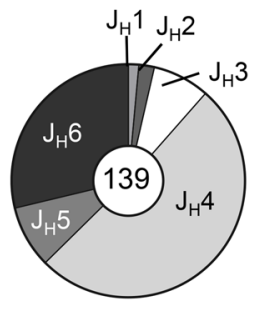

Non-self

+ unknown
$\mathrm{F}+\mathrm{S}$

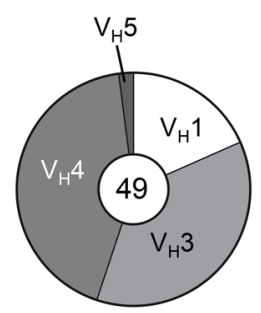

$\mathrm{D}_{\mathrm{H}} 1$
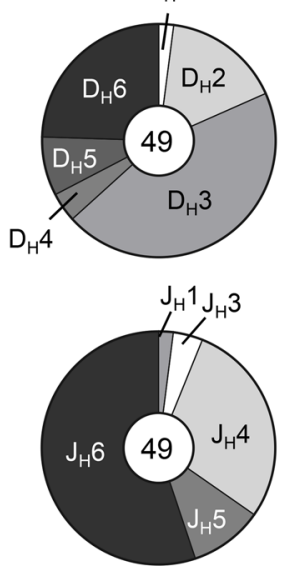
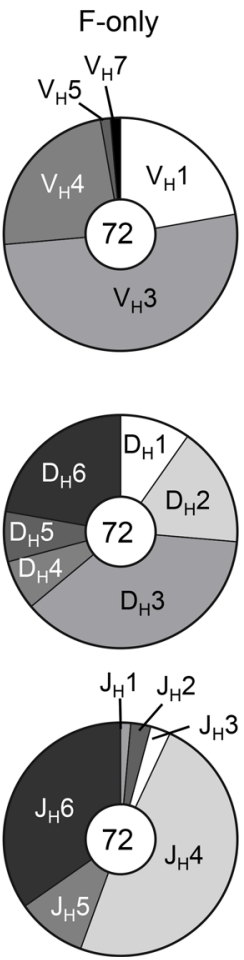

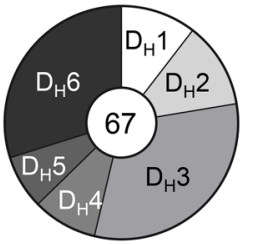

Unknown
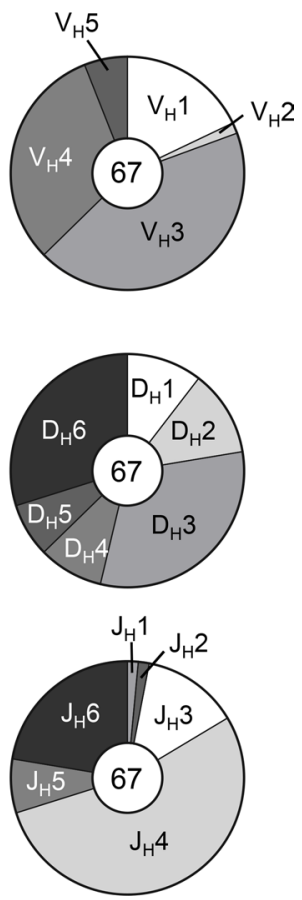

Figure 6. Self-reactive Abs exhibit long HCDR3s with frequent $J_{H} 6$ usage. $V_{H} D J_{H}$ rearrangements were recovered from transitional and mature $B$ cells after single B cell culture. Based on the reactivity of the culture supernatant Abs, samples were grouped as follows: S-only-reactive (filled circles, $n=42$ ); F+S-reactive (filled triangles, $n=49$ ); F-only-reactive (open circles, $n=72$ ); and unknown (open triangles, $n=67$ ). Self-reactive Abs in this experiment represent Abs that reacted with RNP and/or Sm protein. (A) HCDR3 lengths are shown. Each symbol represents an individual sample. ${ }^{* * *} P\langle 0.001, \mathrm{NS}: P>$ 0.05 determined by Mann-Whitney $U$ test. (B) $V_{H}$, D, and $J_{H}$ gene usages are shown. (C) The $J_{H} 6$ usage was compared between self-reactive (S-only-reactive plus $\mathrm{F}+\mathrm{S}, n=91$ ) and non-self ( $\mathrm{F}$-only-reactive plus unknown, $n=139$ ) groups. ${ }^{* *} P<0.01$ determined by $\chi^{2}$ test.

The Ab5 rIgG was cloned from mature, naive B cells of an SLE donor bound to a head-associated HA epitope of influenza H3 Wisconsin (HA/Wis) and human self-antigens, including RNP, dsDNA, Jo-1, Sm, HEp-2 NA, histone, Scl-70, SSB, and centromere B (Supplemental Figure 4). Free HA/Wis-h inhibited Ab5 binding to both HA/Wis and HA/Wis-h on Luminex beads in a dose-dependent manner; the highest concentration of free HA/Wis-h inhibited the binding by $54 \%$ and $71 \%$, respectively, while there was no inhibition by BSA (Figure 7A). Similarly, free HA/Wis-h inhibited binding of Ab5 to RNP and dsDNA by 50\%, while it only modestly (39\%-49\%) inhibited binding to Jo-1, Sm, Hep-2 NA, histone, and Scl-70, and did not inhibit 
binding to SSB and centromere B (Figure 7A). We obtained similar results for other examples of F+S-reactive IgGs, designated as Ab6 and Ab9, respectively, which were cloned from mature, naive $\mathrm{B}$ cells of independent SLE donors (Figure 7B and Supplemental Figures 5-7). Ab6 bound to the head-only construct of influenza HA of H3 Johannesburg (HA/Joburg-h) and self-antigens, including RNP, HEp-2 NA, Sm, centromere B, Scl-70, dsDNA, and histone (Supplemental Figure 5). Free HA/Joburg-h inhibited Ab6 binding to HA/ Joburg by $65 \%$ and to RNP, Hep-2 NA, Sm, and centromere B by $52 \%-64 \%$, respectively (Figure $7 \mathrm{~B}$ ). It modestly inhibited binding to Scl-70 and dsDNA ( $42 \%$ and $39 \%$, respectively) and did not inhibit binding to histone (Figure 7B). Similarly, the highest concentration of free HA/B/Phuket inhibited Ab9 binding to HA/B/Phuket, insulin, and HEp-2 NA by $>50 \%$, and to UBE3A, Sm, Scl-70, and Histone by $32 \%-49 \%$ (Supplemental Figure 7). In all 3 cases, soluble foreign antigens inhibited binding of F+S-reactive Abs to respective foreign antigens and to some self-antigens. We conclude that a class of F+S-reactive BCRs can bind self- and foreign antigens at the same or proximal BCR paratopic site(s) that are close enough for steric hindrance to interfere with antigen binding and that these paratopes are normally made infrequent by tolerance.

\section{Discussion}

Tolerance checkpoints suppress the frequencies and avidities of self-reactive B cells (1, 2, 5, 34). While crucial for physiological homeostasis, by definition, tolerance reduces BCR diversity, in some cases creating "holes" in the B cell repertoire that can be exploited by microbial pathogens. Studies of the immune responses to infections with pathogenic microorganisms, such as C. jejuni and HIV-1, have suggested the importance of the "missing" B cell repertoire within these holes. For example, Abs against oligosaccharides of $C$. jejuni are protective but frequently react with mammalian gangliosides (10), and many HIV-1 bNAbs are either poly- or self-reactive $(11,13,35)$, and this auto- or polyreactivity is associated with the infrequency of protective antibody responses after immunization with $C$. jejuni or HIV-1 antigens, respectively $(10,13)$. This association has led to the "tolerance hypothesis" to explain the low immunogenicity of certain microbial antigens, in which tolerance removes or inactivates B cells capable of generating protective antibody responses due to their recognition of both self- and foreign determinants $(11,15)$.

The question of how great the overlap is between self- and foreign epitopes is an old one (36, 37). Some overlap must exist, as the fundamental structural features of proteins, carbohydrates, lipids, and nucleic acids are common to all life-forms. On the other hand, as the degree of overlap between foreign and self-epitopes increases, BCR diversity among the B cells that persist beyond the tolerance checkpoints must decline; if the 2 sets of epitopes are identical, no B cell could mature, absent failures of tolerance. This problem of discrimination between self and not-self is a core issue of immunobiology but one that can be reasonably ignored, given that humoral immunity to most pathogens is robust and unlinked to autoimmunity. Unusual examples such as the poor humoral protection associated with infection by $C$. jejuni or HIV-1 were just that, unusual.

In this study, we have addressed the issue of self/not-self epitopes in systematic screens of the human $\mathrm{BCR}$ repertoire before and after the second tolerance checkpoint using a Nojima culture system that supports proliferation, differentiation, and IgG secretion of single human transitional and mature B cells (16, 17). In total, we obtained some 2300 clonal IgGs from individual transitional and mature B cells from 14 SLE patients and 9 healthy donors. Taking advantage of a bead-based multiplex assay for IgG binding, we screened these clonal IgG Abs for reactivity to 21 antigens, including 12 self-antigens and 9 foreign antigens; this screening array and set of cloned B cells provided us with $>48,300$ individual data points for analysis. In addition, we obtained $V_{H} D J_{H}$ sequences from selected samples to analyze the somatic genetics of these $\mathrm{B}$ cell populations before and after selection at the second tolerance checkpoint.

In agreement with single B cell studies in healthy humans $(1,2)$, the frequency of self-reactive BCRs/ clonal IgGs in Nojima cultures decreased by half as transitional B cells in the peripheral blood completed their maturation (Figure 1). Given that the frequency of F+S-reactive BCRs also decreased by half during this maturation (Figure 2), peripheral tolerance shapes the BCR repertoire, which is capable of recognizing epitopes on foreign antigens by removing $\mathrm{F}+\mathrm{S}$-reactive $\mathrm{B}$ cells. This is, we think, a crucial point: the second checkpoint removes not some minor component of the BCR repertoire directed to foreign antigens, but almost half of it (Figure 2). Despite the efficacy of the second checkpoint, some $15 \%$ of the clonal IgGs produced by mature $\mathrm{B}$ cells bound self-antigens, and half of these also bound one or more of the 9 foreign antigens in the screen at levels significantly above background (Figure 2). Incomplete deletion and retention of S-only and F+S-reactive $\mathrm{B}$ cells in the mature compartments may contribute to a broader spectrum of BCR specificities and the capacity to respond to microbial pathogens that display antigenic determinants that resemble self $(9,11,38)$. 
A

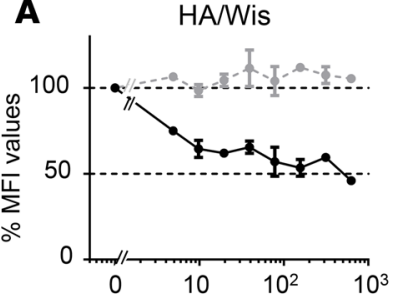

Free H3/Wis-h or BSA $(\mu \mathrm{g} / \mathrm{ml})$

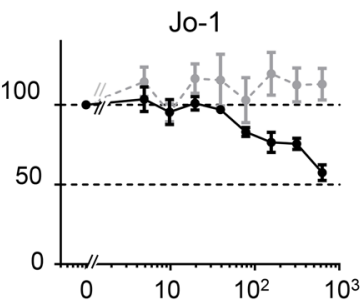

Scl-70
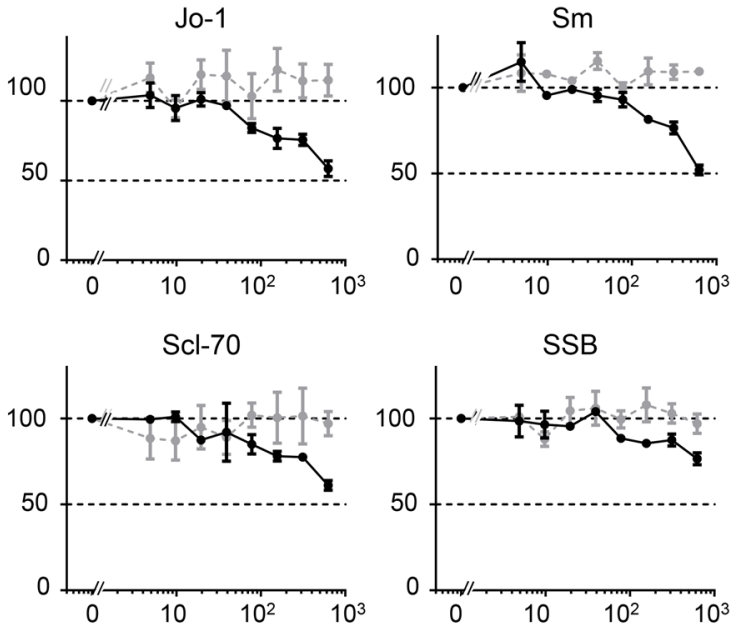

SSB

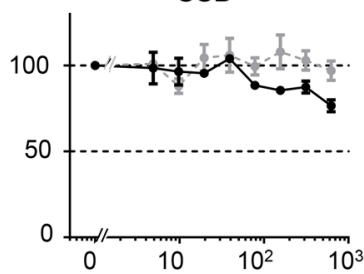

RNP

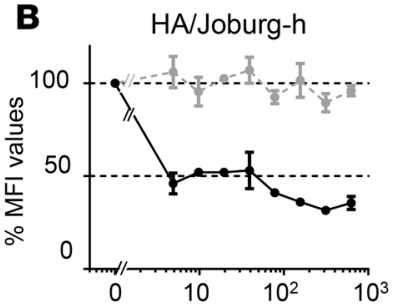

Free H3/Joburg-h or BSA $(\mu \mathrm{g} / \mathrm{ml})$

Centromere B
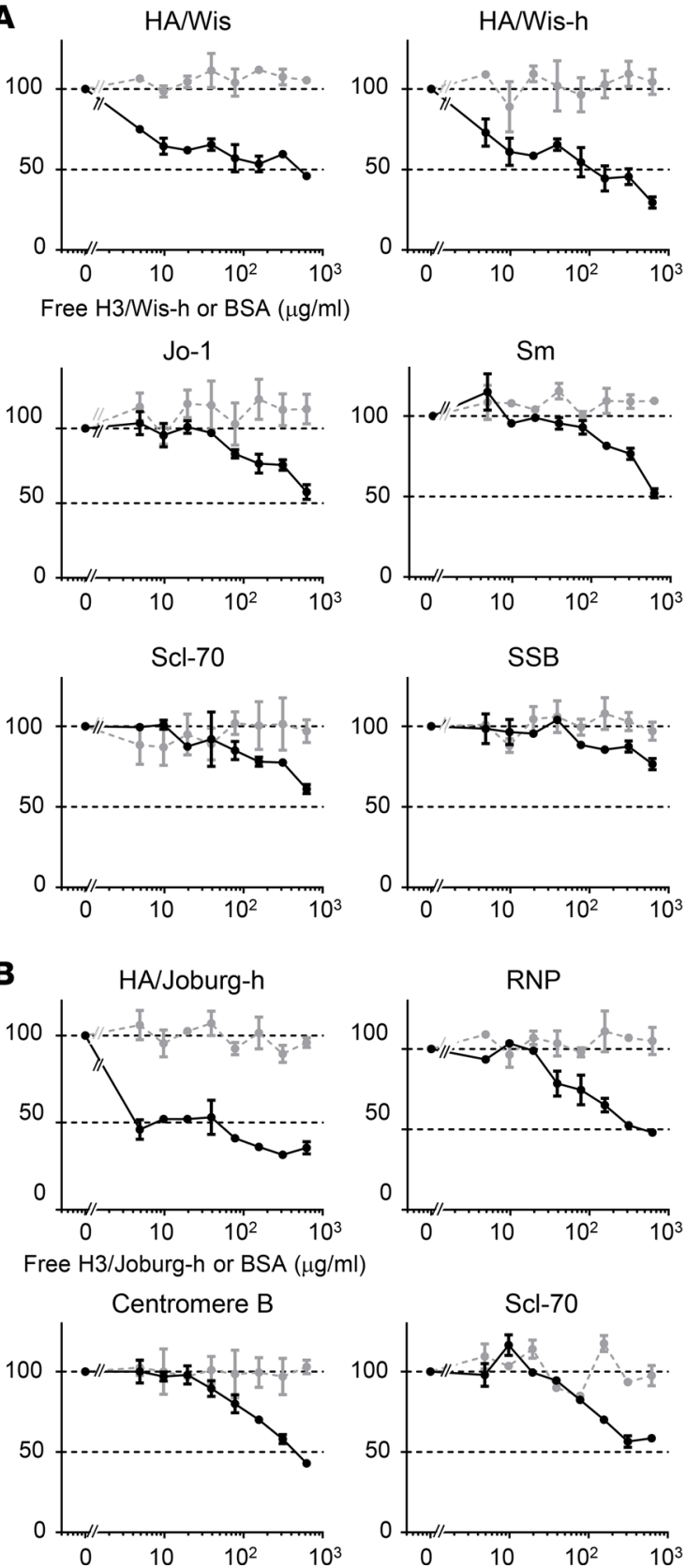
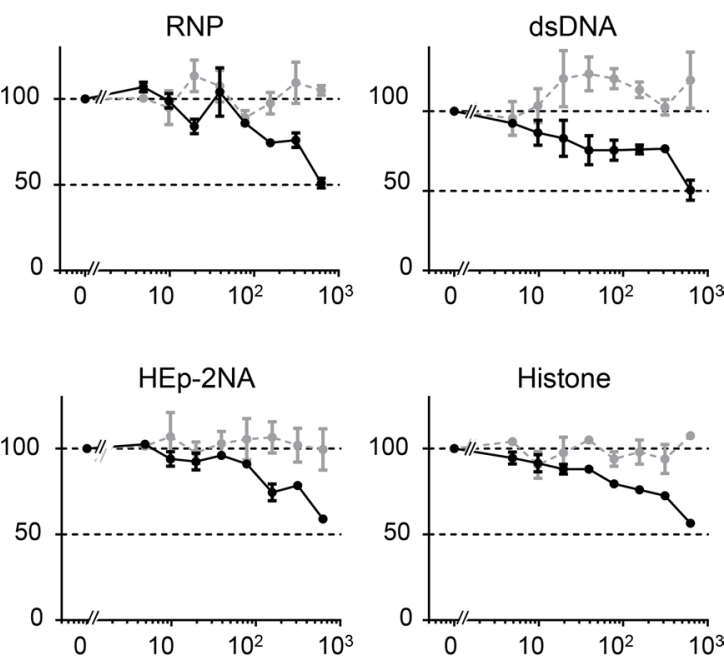

Centromere B

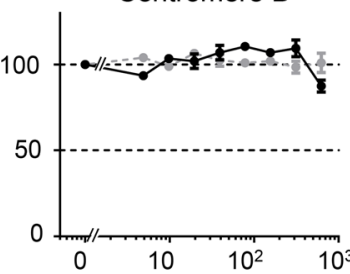

HEp-2NA
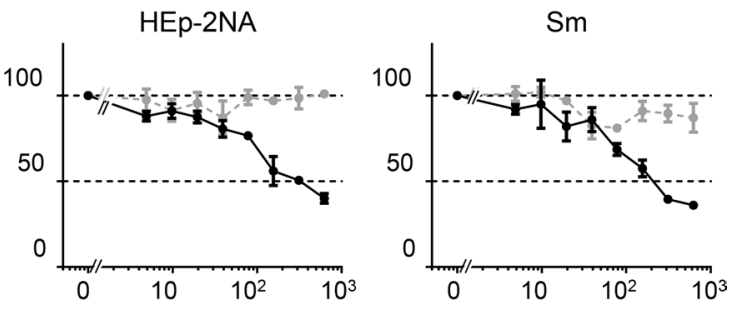

Figure 7. Inhibition of F+S-reactive IgGs by free foreign antigens. Selected F+S-reactive lgGs were incubated with various concentrations of free, either specific or irrelevant (BSA) foreign antigens, and then binding to foreign antigens (homologous inhibition) and self-antigens (heterologous inhibition) was assessed by Luminex multiplex assay. Fixed concentrations of F+S-reactive lgGs (100 $\mu \mathrm{g} / \mathrm{ml}$ for Ab5 in A, $500 \mu \mathrm{g} / \mathrm{ml}$ for Ab6 in B) were first incubated with the indicated concentrations of free HA/Wis-h (A, black lines) or HA/Joburg-h (B, black lines) or BSA (A and $\mathbf{B}$, gray lines), and then these mixtures were added to a panel of antigen-bead conjugates. MFI values for each antigen-bead conjugate were normalized with those without free antigens that were set as $100 \%$. Ab5 bound HA/Wis and HA/Wis-h, and human self-antigens, including RNP, dsDNA, Jo-1, Sm, HEp-2 NA, histone, Scl-70, SSB, and centromere B (Supplemental Figure 4). Ab6 bound HA/Joburg-h and self-antigens, including RNP, HEp-2NA, Sm, centromere B, Scl-70, dsDNA, and histone (Supplemental Figure 5).

In SLE donors, frequencies of self-reactive BCRs/clonal IgGs were unchanged between the transitional and mature B cell compartments (Figure 3), consistent with defective peripheral tolerance in these patients $(2,6)$. The impaired peripheral tolerance checkpoint was accompanied by inefficient removal of F+S-reactive BCRs expressed by B cells in SLE patients (Figure 3 and Supplemental Figure 2). Our results are in contrast to a recent report by Malkiel et al. (39) stating that peripheral tolerance is normal in lupus patients. Although we cannot resolve this discrepancy, methodological differences between studies to assess self-reactivity of B cells may contribute. Malkiel et al. used a flow cytometry-based assay to 
measure B cell binding to nuclear extracts from HeLa cells (39), whereas we and others have used ELISA- or immunofluorescence- $(6,40)$ or multiplex bead-based assays (ref. 17; Figure 1 and Supplemental Figure 2) to define autoreactive IgG binding to HEp-2 cells or purified human self-proteins.

The difference in the $\mathrm{BCR}$ repertoires of mature $\mathrm{B}$ cells from healthy controls and lupus patients is noteworthy. Clonal IgGs produced by mature B cells from SLE donors were significantly more frequently autoreactive than those from healthy individuals and showed a pattern of skewed binding to certain self-antigens (Figure 5). Mature B cells from SLE donors also bound more foreign antigens than did those from healthy controls. These differences in the mature BCR repertoire are, at least in part, due to the absence of reduction in the numbers of $\mathrm{F}+\mathrm{S}$-reactive B cells in SLE patients during the maturation of translational to mature B cells (Figure 3 and Supplemental Figure 2). To our surprise, BCR specificities that were lost to the second tolerance checkpoint included not just those reactive with foreign antigens commonly associated with poor humoral responses such as HIV-1 envelope proteins (41) but also influenza HAs that induce robust antibody responses $(17,42)$ (Figure 4). Can these losses of BCR diversity to tolerance be linked to significant changes in humoral immunity?

Remarkably, many SLE patients develop influenza antibody responses after vaccination that exhibit higher avidity and neutralization capacity than those of matched healthy controls (28). Similarly, a broadly neutralizing recombinant IgG HIV-1 antibody recovered from a rare HIV-1 ${ }^{+}$SLE patient exhibited F+S reactivity by avidly binding to DNA (27). Ineffective deletion of self-reactive mature B cells appears to provide differential and additional foreign specificities that may result in stronger and broader protection against infectious agents in individuals that exhibit impairments of the second tolerance checkpoint $(14,15,27,28)$.

What kind of BCR specificities to foreign epitopes and antigens are contained within the $\mathrm{F}+\mathrm{S}$ compartment of transitional B cells? One set is those BCRs that recognize foreign epitopes that fortuitously mimic those on self-antigens. Examples of this set include the 2F5 broadly neutralizing HIV-1 antibody, which reacts with a rare peptide motif, ELDKWA, shared by the membrane proximal external region of HIV-1 envelope and human KYNU (13); the HIV-1 neutralizing antibody CH98, which also binds double stranded DNA (27); and IgG Abs to $C$. jejuni lipooligosaccharides, which can recognize mammalian gangliosides and other glycans $(10,43)$. In the course of this study, we found that the influenza neutralizing antibody HC19 (44) significantly binds to RNP but not to other autoantigens at concentrations as low as $0.1 \mu \mathrm{g} / \mathrm{ml}$ (Supplemental Figure 8). The HC19 antibody recognizes the HA receptor-binding site, and the structure of HC19 complexed with HA has been solved at high resolution (44). Although HC19 binds HA with much higher avidity than RNP $\left(\approx 5 \times 10^{3}\right.$-fold; Supplemental Figure 8$)$, we think that it represents another example of an F+S-reactive B cell with demonstrated immunological significance (45).

Foreign specificity associated with polyreactivity is also present in the self-reactive compartment but is made infrequent by tolerance. For example, knockin mice with intact tolerance checkpoints expressing BCR of the polyreactive $4 \mathrm{E} 10 \mathrm{HIV}-1 \mathrm{bNAb}$ (35) exhibit impaired B cell development. A class of broadly neutralizing influenza HA stem and receptor binding site Abs also exhibit polyreactivity to human self-antigens (45). Given that $\mathrm{F}+\mathrm{S}$-reactive $\mathrm{B}$ cells bind to 2 or more antigens, they are indeed polyreactive. Regardless of $\mathrm{B}$ cell types (i.e., transitional or mature, naive) or donor types (healthy donors or lupus patients), most (74\%-83\%) F+S-reactive B cells bound multiple foreign and/or self-antigens, respectively, while some $20 \%$ (14\%-25\%) of F-only- or S-only-reactive B cells did. We note, however, that polyreactivity of F+S-reactive $\mathrm{B}$ cells did not uniformly extend to the several control antigens screened, including OVA and KLH (Figure 1, Supplemental Figure 2, and Supplemental Table 1). Therefore, F+S-reactive B cells are not just polyreactive, but comprise BCRs that bind selectively with sufficient avidity to drive B cell activation (46-48). This pattern of "restricted polyreactivity" is exemplified by the HC19 influenza antibody's reactivity solely to RNP (Supplemental Figure 8). Self- and polyreactive human B cells with long HCDR3s commonly use $J_{H} 6$ (Figure 6) but also significantly contribute to a class of broadly neutralizing HIV-1 and influenza Abs that use extended HCDR3 loops to interact with conserved viral epitopes, including the CD4-binding pocket of HIV-1 Env and the receptor-binding site of influenza HA (19-22, 49, 50).

BCR avidities of the clonal IgGs and rIgGs described in our study are undoubtedly low (relative to the K03.12 broadly neutralizing HA antibody [ref. 17], the avidities of $\mathrm{rAb5}$ and $\mathrm{rAb} 6$ range from $<0.01 \%$ to $30 \%$ ). Although we do not fully understand the biological importance of such low-avidity BCRs, their ability to bind in bead assays as bivalent IgG proteins implies that they represent B cells fully capable of entering and participating in T cell-dependent humoral responses $(24,46-48)$. Consistent with this idea, studies have shown that a class of bNAbs to influenza or HIV-1 recovered from memory B cells or plasmablasts or from a phage display library do not bind their nominal antigens when these Abs are expressed as reverted, unmutated germline type (51-57). 
These results suggest that antigen-specific, naive B cells expressing unmeasurably low-avidity BCRs can react with antigens and enter into germinal centers to acquire $V(D) J$ somatic hypermutation to eventually express mutated, high-affinity BCRs/Abs that exhibit biologically important functions.

In humans, the second tolerance checkpoint reduces the frequency of BCRs that bind foreign antigens by almost 50\%, a value comparable to the reduction in BCRs that are autoreactive (Figures 1 and 2). This reduction is largely due to the loss of F+S-reactive BCRs (Figure 2). Given the critical protective role for antibody to many microbial pathogens, and the $\mathrm{F}+\mathrm{S}$ character of many broadly protective Abs to viruses and bacteria, the physiological deletion of F+S-reactive BCRs may significantly limit humoral immunity to microbial pathogens. Moreover, this limitation is not confined to a few emerging or uncommon pathogens, but can be observed prevalent microbial infections, including $C$. jejuni, influenza, and HIV. Whether by chance or selection, microbial epitopes that resemble self-determinants may provide a fitness benefit to pathogens, by slowing their clearance and increasing the likelihood of transmission. It may be a useful vaccine strategy to activate $\mathrm{F}+\mathrm{S}$-reactive B cell populations normally removed or made infrequent by tolerance by the transient modulation of central or peripheral tolerance checkpoints in ways that maximize antibody responses to vaccines but minimize the risk of autoimmune disease. At present it is possible to manipulate tolerance checkpoints by treatment with chloroquine (34) or inhibitors of immune regulation (15) to expand the B cell repertoire against foreign antigens, but this comes with increased risk of autoimmune disorder. Reciprocally, whereas increased stringency at tolerance checkpoints would minimize autoimmunity, it would also be likely to reduce humoral responses to many foreign pathogens. Understanding the balanced regulation of immunological tolerance and the role of $\mathrm{F}+\mathrm{S}$-reactive $\mathrm{B}$ cells in protective immunity is an important goal for designing effective vaccination strategies against microbial pathogens, and especially those that benefit from host mimicry.

\section{Methods}

Patient samples. This study was performed in accordance with Duke Institutional Review Board committee guidelines, and all samples were obtained after receipt of signed informed consent. PBMC samples from 14 SLE patients and 5 healthy controls were provided in a blinded fashion by the Immune Tolerance Network. Peripheral blood samples from 4 additional healthy controls were obtained from subjects recruited at Duke University Medical Center. The SLE samples came from subjects undergoing screening for the ACCESS study (a 1:1 randomized, double-blind, controlled phase II multicenter trial of abatacept [ABA] versus placebo on a background of treatment with glucocorticoid [GC] plus cyclophosphamide [CTX] followed by azathioprine [AZA] in patients with active lupus nephritis). The ACCESS study was sponsored by the National Institute of Allergy and Infectious Diseases (NIAID) and conducted by the Immune Tolerance Network (ITN034AI) (ClinicalTrials.gov NCT00774852). The protocol was reviewed and approved by the IRB at each of the clinical sites, and all subjects provided informed consent prior to their participation. Samples from 12 female and 2 male subjects with SLE that had a mean \pm SD age of $27.6 \pm 9.6$ years and a mean $\pm \mathrm{SD}$ duration of disease of $71 \pm 59$ months were included. Among the lupus samples, 14 of 14 tested positive for antinuclear Abs; 11 of 14 tested positive for Abs to dsDNA; 6 of 13 tested positive for anti-RNP Abs; 3 of 13 tested positive for anti-Sm Abs; and 1 of 13 tested positive for IgG anti-cardiolipin Abs. At the time of sample acquisition, subjects were taking a variety of immunomodulatory drugs, including prednisone (13 of 14 subjects, mean \pm SD daily dose of $43 \pm 20 \mathrm{mg}$ ), mycophenolate mofetil (5 of 14 subjects), and hydroxychloroquine (5 of 14 subjects). One of the subjects taking oral prednisone had also been receiving $500 \mathrm{mg}$ methylprednisolone i.v every day for 3 days. Mononuclear leukocytes were isolated from blood samples by centrifugation over Ficoll density gradients (SepMate-50 tubes, STEMCELL Technologies). Cell samples were frozen and kept in liquid nitrogen until use.

Flow cytometry and definition of transitional and mature B cells. Transitional and mature B cell isolations were performed by flow cytometry. Briefly, the cells were labeled with fluorochrome-conjugated mAbs specific for human surface antigens in RPMI containing 10\% FBS. The following mAbs specific for human surface antigens were used. Anti-human IgM-FITC (MHM-88), CD27-PE (L128), CD3-PE-Cy5 (UCHT1), CD14Tri (TuK4), CD16-PE-Cy5 (3G8), CD10-PE-Cy7 (HI10a), CD19-APC (HIB19), IgD-APC-Cy7 (IA6-2), CD38-BV421 (HIT2), and CD24-BV510 (ML5) were purchased from BD Biosciences or BioLegend. Mouse $\mathrm{IgG1 \kappa}$ isotype control (MG1K) was purchased from Rockland. Labeled cells were sorted by FACSAria or FACSVantage with Diva software (BD Biosciences). Flow cytometric data were analyzed with FlowJo software. Doublets were excluded from cell sorting by combinations of FSC-A versus FSC-H gating. 7-AAD-positive (BD Biosciences) cells (dead cells) and CD3/CD14/CD16-positive cells were also excluded. B cell subsets 
were identified as follows: transitional $\mathrm{B}, \mathrm{CD} 19^{+} \mathrm{CD} 27^{-} \mathrm{CD} 38^{\text {hi }} \mathrm{CD} 10^{+} \mathrm{IgD}^{+}$; mature $\mathrm{B}, \mathrm{CD} 19^{+} \mathrm{CD} 27^{-} \mathrm{CD} 38^{\text {hi }} \mathrm{C}$ $\mathrm{D}^{+} 0^{+} \mathrm{IgD}^{+}$cells (23). BD Calibrite Beads (BD Biosciences) were used to count $\mathrm{B}$ cells in cultures.

Single-cell cultures for human B cells. Single transitional B cells and mature B cells from SLE patients and healthy control donors were expanded in the presence of MS40L $\mathrm{L}^{\text {lo }}$ feeder cells, which are transformed murine stromal MS5 cells that provide a low level of anchored CD154 (58). Briefly, single B cells were directly sorted and cultured in each well of 96-well plates with MS40L ${ }^{\text {1o }}$ feeder cells in RPMI 1640-containing (Invitrogen) 10\% HyClone FBS (Thermo Fisher Scientific), 2-mercaptoethanol $\left(5.5 \times 10^{-5} \mathrm{M}\right)$, penicillin $(100 \mathrm{U} / \mathrm{ml})$, streptomycin $(100 \mu \mathrm{g} / \mathrm{ml})$, HEPES $(10 \mathrm{mM})$, sodium pyruvate $(1 \mathrm{mM})$, and MEM nonessential amino acid (1×; all Invitrogen) and exogenous recombinant human IL-2 (50 ng/ml), IL-4 (10 ng/ $\mathrm{ml})$, IL-21 (10 ng/ml), and BAFF (10 ng/ml; all Peprotech). Half of the culture media was replaced with fresh media twice each week. On day 25 , culture supernatants were harvested for screening the reactivity of clonal IgG Abs. Expanded clonal B cells were frozen for sequence analyses.

Multiplex bead Luminex assay. To screen the reactivity of clonal IgG Abs in culture supernatants for their specificity for a panel of self- and foreign antigens, a multiplex bead Luminex assay was performed according to the manufacturer's protocol (Luminex Corp.). The AtheNA Multi-Lyte ANA-II Plus Test System (Zeus Scientific) was used for determination of autoantibodies specific for 10 different antigens (SSA, SSB, Sm, RNP, Scl-70, Jo-1, centromere B, histone, dsDNA, and HEp-2 NA). The following antigens were coupled to carboxylated beads (Luminex Corp.): insulin (MilliporeSigma), KYNU (13), HIV-1 gp41, gp140 JR-FL (provided by the Duke Human Vaccine Institute; ref. 59), NP-BSA (coupled in-house), OVA (MilliporeSigma), KLH (MilliporeSigma), rPA (BEI Resources), SA (Invitrogen), influenza HAs, including H1 A/Solomon Islands/03/2006 (H1 SI), H1 A/California/04/2009 (H1 CA), H1 A/reassortant/ NYMC X-181(California/07/2009 3 NYMC X-157) (X181), H3 A/Aichi/2/1968 (X31) (H3 X31), H5 A/Vietnam/1203/2004 (H5 VN), and B/Malaysia/2506/2004 (B Malaysia); and trimeric, head-only rHA constructs of H3 A/Wisconsin/67/2005 (H3 Wis-tri-h) and H3 A/Johannesburg/33/1994 (H3 Joburgtri-h) (generated in-house; refs. 21, 49), goat anti-human Igк, Ig $\lambda$ (both Southern Biotech), and anti-human IgG (Jackson ImmunoResearch Laboratories Inc.). Briefly, diluted culture supernatants (at 1:10 dilutions in assay buffer: $1 \times$ PBS containing $1 \%$ milk, $1 \%$ BSA, $0.05 \% \mathrm{NaN}_{3}$, and $0.05 \%$ Tween-20) and serially diluted standard anti-human dsDNA polyclonal Abs (LSBio) were incubated with the mixture of microspheres in a 96-well filter-bottom plate (Millipore). After incubation for 2 hours, the beads were washed by vacuum filtration 3 times and further incubated for 1 hour with PE-conjugated goat anti-human IgG Abs supplied by the AtheNA Multi-Lyte ANA-II Plus Test System. After 3 washes, the beads were resuspended with assay buffer, and the plates were read on the Bio-Plex 3D Suspension Array System (Bio-Rad). Culture supernatants that contained total IgGs at $1-270 \mu \mathrm{g} / \mathrm{ml}$ were used for determination of antigen binding. Binding to BSA was considered as nonspecific binding, and those samples were not counted for antigen reactivity.

Amplification of $V_{H} D J_{H}$ rearrangements. Nested PCR was performed to amplify human $V_{H} D J_{H}$ rearrangements from cDNA samples of cultured B cells. Briefly, total RNA was extracted from selected samples using TRIzol reagent (Invitrogen). cDNA was synthesized from DNase I-treated RNA using Superscript III with oligo- $\mathrm{dT}_{20}$ primers. One-twentieth (volume) of the $\mathrm{cDNA}$ was then subjected to 2 rounds of PCR using Herculase II fusion DNA polymerase (Agilent Technologies) with established primers (60-62). PCR conditions (both primary and secondary PCR) were $94^{\circ} \mathrm{C}$ for 4 minutes, followed by 30 (for primary) or 40 cycles (for secondary) at $94^{\circ} \mathrm{C}$ for 30 seconds, $58^{\circ} \mathrm{C}$ for 30 seconds, and $72^{\circ} \mathrm{C}$ for 45 seconds. $\mathrm{V}_{\mathrm{H}} \mathrm{DJ}_{\mathrm{H}}$ amplicands were gel purified, ligated into vectors, and transformed into bacteria as previously described (63). DNA sequences were obtained at the Duke University DNA Analysis Facility. $\mathrm{V}_{\mathrm{H}} \mathrm{DJ}_{\mathrm{H}}$ rearrangements were identified with IMGT/V-QUEST (http://www.imgt.org/IMGT_vquest/vquest).

Recombinant IgG expression. From selected samples, we reconstituted IgG Abs (24, 62). Briefly, cloned $\mathrm{V}(\mathrm{D}) \mathrm{J}$ rearrangements were ligated into $\mathrm{IgH}$ and $\mathrm{IgL}$ expression vectors (gift of $\mathrm{H}$. Wardemann, German Cancer Research Center, Heidelberg, Germany), and then these expression vectors were cotransfected into HEK293 cells. Recombinant Abs were purified from the culture supernatants using NAb protein G spin columns (Thermo Fisher Scientific) and used for subsequent assays.

Inhibition assay. Competitive inhibition assays with $\mathrm{rAbs}$ from the $\mathrm{F}+\mathrm{S}$-reactive group were performed in Luminex multiplex assay. Fixed concentrations of rAbs were incubated with serial dilutions of free HAs or BSA for overnight at $4^{\circ} \mathrm{C}$. After incubation, these mixtures (rAbs and free proteins) were further incubated with the mixture of microspheres, followed by incubation with PE-conjugated goat anti-human IgG Abs (SouthernBiotech). Plates were read on the Bio-Plex 3D Suspension Array System. 
Statistics. Statistical significance $(P<0.05)$ was determined by Wilcoxon matched-pairs signed-rank test (2-tailed), Mann-Whitney's $U$ test, or the $\chi^{2}$ independence test. Statistical analysis was performed using GraphPad Prism 6 (GraphPad Software).

Study approval. This study was performed in accordance with and approved by Duke Institutional Review Board committee guidelines, and all samples were obtained after receipt of signed informed consent. PBMC samples from 14 patients with SLE and 5 healthy control individuals were provided in a blinded fashion by the Immune Tolerance Network. Peripheral blood samples from 4 additional healthy controls were obtained from subjects recruited at the Duke University Medical Center. The SLE samples came from subjects undergoing screening for the ACCESS study (a 1:1 randomized, double-blind, controlled phase II multicenter trial of ABA versus placebo on a background of treatment with GC plus CTX followed by AZA in patients with active lupus nephritis). The ACCESS study was sponsored by theNIAID and conducted by the Immune Tolerance Network (ITN034AI) (ClinicalTrials.gov NCT00774852). The protocol was reviewed and approved by the IRB at each of the clinical sites, and all subjects provided informed consent prior to participation.

\section{Author contributions}

AW, MK, and GK designed experiments. AW and MK conducted experiments. AW, MK, and GK analyzed results. AW and KYS established human single B cell cultures. AW and GY established Luminex multiplex assays. AGS and SCH provided reagents. AW, MK, AER, SCH, EWS, and GK wrote the manuscript. BFH, EWS, and GK conceived the study. GK supervised the study.

\section{Acknowledgments}

Research specimens from the ITN034AI ACCESS trial reported in this publication were provided by the Immune Tolerance Network and supported by the NIAID of the NIH under award UM1AI109565. The content is solely the responsibility of the authors and does not necessarily represent the official views of the NIH. We thank David Baltimore for providing the MS40L ${ }^{10}$ cell line. We thank Goran Bajic for comments on the manuscript. We are grateful for the technical support of Xiaoyan Nie, Xiaoe Liang, Dongmei Liao, and Sergio Sanders. This research was supported in part by Autoimmunity Center of Excellence (ACE) grant U19-AI-056363, NIH grant HHSN272201000053C, and NIH/NIAID grant UM1AI109565.

Address correspondence to: Garnett Kelsoe, Department of Immunology, Duke University, Durham, North Carolina 27710, USA. Phone: 919.613.7815; Email: ghkelsoe@duke.edu.

1. Wardemann H, Yurasov S, Schaefer A, Young JW, Meffre E, Nussenzweig MC. Predominant autoantibody production by early human B cell precursors. Science. 2003;301(5638):1374-1377.

2. Wardemann H, Nussenzweig MC. B-cell self-tolerance in humans. Adv Immunol. 2007;95:83-110.

3. Goodnow CC, Crosbie J, Jorgensen H, Brink RA, Basten A. Induction of self-tolerance in mature peripheral B lymphocytes. Nature. 1989;342(6248):385-391.

4. Russell DM, Dembić Z, Morahan G, Miller JF, Bürki K, Nemazee D. Peripheral deletion of self-reactive B cells. Nature. 1991;354(6351):308-311.

5. Meffre E, Wardemann H. B-cell tolerance checkpoints in health and autoimmunity. Curr Opin Immunol. 2008;20(6):632-638.

6. Yurasov S, et al. Defective B cell tolerance checkpoints in systemic lupus erythematosus. J Exp Med. 2005;201(5):703-711.

7. Samuels J, Ng YS, Coupillaud C, Paget D, Meffre E. Impaired early B cell tolerance in patients with rheumatoid arthritis. $J$ Exp Med. 2005;201(10):1659-1667.

8. Meffre E. The establishment of early B cell tolerance in humans: lessons from primary immunodeficiency diseases. Ann $N Y$ Acad Sci. 2011;1246:1-10.

9. Goodnow CC. Balancing immunity and tolerance: deleting and tuning lymphocyte repertoires. Proc Natl Acad Sci USA. 1996;93(6):2264-2271.

10. Bowes T, et al. Tolerance to self gangliosides is the major factor restricting the antibody response to lipopolysaccharide core oligosaccharides in Campylobacter jejuni strains associated with Guillain-Barré syndrome. Infect Immun. 2002;70(9):5008-5018.

11. Haynes BF, et al. Cardiolipin polyspecific autoreactivity in two broadly neutralizing HIV-1 antibodies. Science. 2005;308(5730):1906-1908.

12. Haynes BF, et al. HIV-host interactions: implications for vaccine design. Cell Host Microbe. 2016;19(3):292-303.

13. Yang G, et al. Identification of autoantigens recognized by the $2 \mathrm{~F} 5$ and $4 \mathrm{E} 10$ broadly neutralizing HIV-1 antibodies. J Exp Med. 2013;210(2):241-256.

14. Haynes BF, Moody MA, Verkoczy L, Kelsoe G, Alam SM. Antibody polyspecificity and neutralization of HIV-1: a hypothesis. Hum Antibodies. 2005;14(3-4):59-67.

15. Kelsoe G, Verkoczy L, Haynes BF. Immune system regulation in the induction of broadly neutralizing HIV-1 antibodies. Vaccines (Basel). 2014;2(1):1-14. 
16. Su KY, Watanabe A, Yeh CH, Kelsoe G, Kuraoka M. Efficient culture of human naive and memory B cells for use as APCs. J Immunol. 2016;197(10):4163-4176.

17. McCarthy KR, et al. Memory B cells that cross-react with group 1 and group 2 influenza A viruses are abundant in adult human repertoires. Immunity. 2018;48(1):174-184.e9.

18. Schmidt AG, et al. Viral receptor-binding site antibodies with diverse germline origins. Cell. 2015;161(5):1026-1034.

19. Saphire EO, et al. Crystal structure of a neutralizing human IGG against HIV-1: a template for vaccine design. Science. 2001;293(5532):1155-1159.

20. Pejchal R, et al. Structure and function of broadly reactive antibody PG16 reveal an H3 subdomain that mediates potent neutralization of HIV-1. Proc Natl Acad Sci USA. 2010;107(25):11483-11488.

21. Whittle JR, et al. Broadly neutralizing human antibody that recognizes the receptor-binding pocket of influenza virus hemagglutinin. Proc Natl Acad Sci USA. 2011;108(34):14216-14221.

22. Ekiert DC, et al. Cross-neutralization of influenza A viruses mediated by a single antibody loop. Nature. 2012;489(7417):526-532

23. Carsetti R, Rosado MM, Wardmann H. Peripheral development of B cells in mouse and man. Immunol Rev. 2004;197:179-191.

24. Kuraoka M, et al. Complex antigens drive permissive clonal selection in germinal centers. Immunity. 2016;44(3):542-552.

25. Verkoczy L, et al. Autoreactivity in an HIV-1 broadly reactive neutralizing antibody variable region heavy chain induces immunologic tolerance. Proc Natl Acad Sci USA. 2010;107(1):181-186.

26. Chen Y, et al. Common tolerance mechanisms, but distinct cross-reactivities associated with gp41 and lipids, limit production of HIV-1 broad neutralizing antibodies 2F5 and 4E10. J Immunol. 2013;191(3):1260-1275.

27. Bonsignori $\mathrm{M}$, et al. An autoreactive antibody from an SLE/HIV-1 individual broadly neutralizes HIV-1. J Clin Invest. 2014;124(4):1835-1843.

28. Kaur K, et al. High Affinity Antibodies against influenza characterize the plasmablast response in SLE patients after vaccination. PLoS ONE. 2015;10(5):e0125618.

29. Tápanes FJ, Vásquez M, Ramírez R, Matheus C, Rodríguez MA, Bianco N. Cluster analysis of antinuclear autoantibodies in the prognosis of SLE nephropathy: are anti-extractable nuclear antibodies protective? Lupus. 2000;9(6):437-444.

30. Migliorini P, Baldini C, Rocchi V, Bombardieri S. Anti-Sm and anti-RNP antibodies. Autoimmunity. 2005;38(1):47-54.

31. Ichiyoshi Y, Casali P. Analysis of the structural correlates for antibody polyreactivity by multiple reassortments of chimeric human immunoglobulin heavy and light chain V segments. J Exp Med. 1994;180(3):885-895.

32. Crouzier R, Martin T, Pasquali JL. Heavy chain variable region, light chain variable region, and heavy chain CDR3 influences on the mono- and polyreactivity and on the affinity of human monoclonal rheumatoid factors. J Immunol. 1995;154(9):4526-4535.

33. Aguilera I, Melero J, Nuñez-Roldan A, Sanchez B. Molecular structure of eight human autoreactive monoclonal antibodies. Immunology. 2001;102(3):273-280.

34. Kuraoka M, et al. BCR and endosomal TLR signals synergize to increase AID expression and establish central B cell tolerance. Cell Rep. 2017;18(7):1627-1635.

35. Liu M, et al. Polyreactivity and autoreactivity among HIV-1 antibodies. J Virol. 2015;89(1):784-798.

36. Cohn M. The wisdom of hindsight. Annu Rev Immunol. 1994;12:1-62.

37. Cohn M. On the opposing views of the self-nonself discrimination by the immune system. Immunol Cell Biol. 2009;87(2):113-119.

38. Zikherman J, Parameswaran R, Weiss A. Endogenous antigen tunes the responsiveness of naive B cells but not T cells. Nature. 2012;489(7414):160-164.

39. Malkiel S, et al. Checkpoints for autoreactive B cells in peripheral blood of lupus patients assessed by flow cytometry. Arthritis Rheumatol. 2016;68(9):2210-2220.

40. Yurasov S, Nussenzweig MC. Regulation of autoreactive antibodies. Curr Opin Rheumatol. 2007;19(5):421-426.

41. Haynes BF, Kelsoe G, Harrison SC, Kepler TB. B-cell-lineage immunogen design in vaccine development with HIV-1 as a case study. Nat Biotechnol. 2012;30(5):423-433.

42. Moody MA, et al. H3N2 influenza infection elicits more cross-reactive and less clonally expanded anti-hemagglutinin antibodies than influenza vaccination. PLoS One. 2011;6(10):e25797.

43. Houliston RS, et al. Lipooligosaccharide of Campylobacter jejuni: similarity with multiple types of mammalian glycans beyond gangliosides. J Biol Chem. 2011;286(14):12361-12370.

44. Bizebard T, et al. Structure of influenza virus haemagglutinin complexed with a neutralizing antibody. Nature. 1995;376(6535):92-94

45. Bajic G, et al. Autoreactivity profiles of influenza hemagglutinin broadly neutralizing antibodies. Sci Rep. 2019;9(1):3492.

46. Dal Porto JM, Haberman AM, Shlomchik MJ, Kelsoe G. Antigen drives very low affinity B cells to become plasmacytes and enter germinal centers. J Immunol. 1998;161(10):5373-5381.

47. Dal Porto JM, Haberman AM, Kelsoe G, Shlomchik MJ. Very low affinity B cells form germinal centers, become memory B cells, and participate in secondary immune responses when higher affinity competition is reduced. J Exp Med. 2002;195(9):1215-1221.

48. Shih TA, Meffre E, Roederer M, Nussenzweig MC. Role of BCR affinity in T cell dependent antibody responses in vivo. Nat Immunol. 2002;3(6):570-575.

49. Schmidt AG, et al. Preconfiguration of the antigen-binding site during affinity maturation of a broadly neutralizing influenza virus antibody. Proc Natl Acad Sci USA. 2013;110(1):264-269.

50. Schmidt AG, et al. Immunogenic stimulus for germline precursors of antibodies that engage the influenza hemagglutinin receptor-binding site. Cell Rep. 2015;13(12):2842-2850.

51. Xiao X, et al. Germline-like predecessors of broadly neutralizing antibodies lack measurable binding to HIV-1 envelope glycoproteins: implications for evasion of immune responses and design of vaccine immunogens. Biochem Biophys Res Commun. 2009;390(3):404-409.

52. Scheid JF, et al. Sequence and structural convergence of broad and potent HIV antibodies that mimic CD4 binding. Science. 2011;333(6049):1633-1637.

53. Lingwood D, et al. Structural and genetic basis for development of broadly neutralizing influenza antibodies. Nature 2012;489(7417):566-570. 
54. Hoot S, et al. Recombinant HIV envelope proteins fail to engage germline versions of anti-CD4bs bNAbs. PLoS Pathog. 2013;9(1):e1003106.

55. McGuire AT, et al. Engineering HIV envelope protein to activate germline B cell receptors of broadly neutralizing anti-CD4 binding site antibodies. J Exp Med. 2013;210(4):655-663.

56. Jardine J, et al. Rational HIV immunogen design to target specific germline B cell receptors. Science. 2013;340(6133):711-716.

57. Zhou T, et al. Structural Repertoire of HIV-1-neutralizing antibodies targeting the CD4 supersite in 14 donors. Cell. 2015;161(6):1280-1292.

58. Luo XM, Maarschalk E, O'Connell RM, Wang P, Yang L, Baltimore D. Engineering human hematopoietic stem/progenitor cells to produce a broadly neutralizing anti-HIV antibody after in vitro maturation to human B lymphocytes. Blood. 2009;113(7):1422-1431.

59. Ma BJ, et al. Envelope deglycosylation enhances antigenicity of HIV-1 gp41 epitopes for both broad neutralizing antibodies and their unmutated ancestor antibodies. PLoS Pathog. 2011;7(9):e1002200.

60. Rohatgi S, Ganju P, Sehgal D. Systematic design and testing of nested (RT-)PCR primers for specific amplification of mouse rearranged/expressed immunoglobulin variable region genes from small number of B cells. J Immunol Methods. 2008;339(2):205-219.

61. Tiller T, Busse CE, Wardemann H. Cloning and expression of murine Ig genes from single B cells. J Immunol Methods. 2009;350(1-2):183-193.

62. Tiller T, Meffre E, Yurasov S, Tsuiji M, Nussenzweig MC, Wardemann H. Efficient generation of monoclonal antibodies from single human B cells by single cell RT-PCR and expression vector cloning. J Immunol Methods. 2008;329(1-2):112-124.

63. McWilliams L, et al. The human fetal lymphocyte lineage: identification by CD27 and LIN28B expression in B cell progenitors. J Leukoc Biol. 2013;94(5):991-1001. 